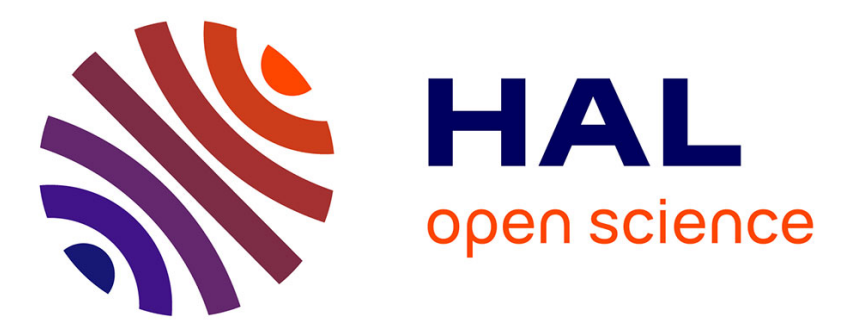

\title{
From steady to unsteady laminar flow in model porous structures: an investigation of the first Hopf bifurcation
}

Mehrez Agnaou, Didier Lasseux, Azita Ahmadi

\section{To cite this version:}

Mehrez Agnaou, Didier Lasseux, Azita Ahmadi. From steady to unsteady laminar flow in model porous structures: an investigation of the first Hopf bifurcation. Computers and Fluids, 2016, 136, pp.67-82. 10.1016/j.compfluid.2016.05.030 . hal-01329941

\section{HAL Id: hal-01329941 \\ https://hal.science/hal-01329941}

Submitted on 9 Jun 2016

HAL is a multi-disciplinary open access archive for the deposit and dissemination of scientific research documents, whether they are published or not. The documents may come from teaching and research institutions in France or abroad, or from public or private research centers.
L'archive ouverte pluridisciplinaire HAL, est destinée au dépôt et à la diffusion de documents scientifiques de niveau recherche, publiés ou non, émanant des établissements d'enseignement et de recherche français ou étrangers, des laboratoires publics ou privés. 


\title{
From steady to unsteady laminar flow in model porous structures: an investigation of the first Hopf bifurcation
}

\author{
M. Agnaou, D. Lasseux*, A. Ahmadi \\ CNRS, Univ. of Bordeaux, IPB, ENSAM - I2M, UMR5295 \\ Esplanade des Arts et Métiers \\ 33405 Talence, Cedex, France
}

\begin{abstract}
This work focuses on the occurrence of the first Hopf bifurcation, corresponding to the transition from steady to unsteady flow conditions, on $2 D$ periodic ordered and disordered non-deformable porous structures. The structures under concern, representative of real systems for many applications, are composed of cylinders of square cross section for values of the porosity ranging from $15 \%$ to $96 \%$. The critical Reynolds number at the bifurcation is determined for incompressible isothermal Newtonian fluid flow by Direct Numerical Simulations (DNS) based on a finite volume discretization method that is second order accurate in space and time. It is shown that for ordered square periodic structures, the critical Reynolds number increases when the porosity decreases and strongly depends on the choice of the Representative Elementary Volume on which periodic boundary conditions are employed. The flow orientation with respect to the principal axes of the structure is also shown to have a very important impact on the value of the Reynolds number of the bifurcation. When structural disorder is introduced, the critical Reynolds number decreases very significantly in comparison to the ordered structure having the same porosity. Correlations between the critical Reynolds number and the porosity are obtained on both ordered and disordered structures over wide range of porosities. A frequency analysis is performed on one of the velocity components to investigate preand post-bifurcation flow characteristics.

Keywords: Porous media, Non-Darcy flow, Unsteady one-phase flow, Critical Reynolds number, Hopf bifurcation
\end{abstract}

\section{Introduction}

Flow through porous media out of the classical creeping or Darcy regime is encountered in a wide range of applications, among which one can cite flow near wells in petroleum engineering, flow in filters and in columns of reactors for chemical engineering, flow through constructs in perfused or agitated bioreactors

\footnotetext{
* Corresponding author

Email address: didier.lasseux@ensam.eu (D. Lasseux )
} 

inertial correction exhibits a distinct dependence upon the filtration velocity. A regime beyond the strong inertia has also been identified based on the inertial correction analysis [23, 24, 25]. 
Inertial flow classification adopted in almost all analyses has been addressed for flow under steady con-

40 corresponds to the Hopf bifurcation in the structures under concern [26], is of major interest and this is the main purpose of the present work. The Hopf bifurcation corresponds to the first bifurcation of the steady flow solution for a large enough (critical) value of the Reynolds number towards a time-periodic solution. Loss of steadiness takes place in vortices and gives rise to an oscillatory flow at the bifurcation. from the macroscopic transport equations by time-averaging, have revealed to be quite inaccurate [28]. As an alternative, models where the microscopic transport equations are first time averaged and then up-scaled have been proposed [29, 30].

So far, most of existing investigations on the Hopf bifurcation have been carried out for flow around a unique cylinder of circular or square cross section or for flow past a row of cylinders.

Flow past a circular cylinder has been analyzed for both the laminar [31] and turbulent [32] flow regimes. Similar work has been dedicated to the case of flow past a square cylinder, first in the unsteady 2D case with a zero angle of incidence [33], and then in 3D, [34,3] considering different angles of incidence [35]. Surprisingly, much less attention has been paid to 2D or 3D patterns of obstacles, the case of a single row of square cylinders being an exception. In that case, experimental studies [36] and later, numerical simulations $[37,38]$ have been carried out that shed light on the physics of this type of flow. A short analysis of the critical Reynolds number at which unsteadiness appears for a periodic regular array of parallel cylinders of circular cross section was reported based on numerical simulations carried out over a narrow range of the fluid volume fraction (i.e. the porosity) with a special focus on the effect of the pressure gradient orientation with respect to the lattice axes of the structure [39]. Recent experiments [40] on floor water inrush have highlighted a qualitative observation showing that the smaller the porosity of the porous medium, the larger the critical value of the Reynolds number at which unsteadiness occurs.

To the best of our knowledge, a detailed analysis of the dependence of the critical Reynolds number corresponding to the first Hopf bifurcation on parameters such as porosity, pressure gradient orientation, disorder of the structure and the size of the Representative Elementary Volume, although of considerable importance, is still lacking and is the objective of the present work.

Two-dimensional arrays of parallel cylinders of square cross section are considered here as a primary generic approach to more complex porous structures as well as a realistic model for many practical situations such as environmental flows mentioned above. The attention is focused on the critical Reynolds number value corresponding to the limit of stationary laminar flow orthogonal to the cylinders axes.

The paper is organized as follows. The initial boundary value problem and the numerical method used for the resolution are presented in section 2. Validation tests operated on the classical case of flow around a 
unique cylinder of square cross section and flow around a cylinder of circular cross section in a square pattern are then reported. In section 3, the dependence of the critical Reynolds number on the Representative Elementary Volume and porosity is presented in the case of an ordered structure (referred to as $O S$ in the following) made of square patterns of parallel cylinders of square cross section. Process leading to unsteady flow is investigated. A spectral analysis of the transverse velocity signal is carried out and the dependence of the critical Reynolds number on the pressure gradient orientation is discussed. Finally the case of disordered structures $(D S)$ obtained from a random placement of the cylinders in each original square unit cell of the $O S[23]$ is considered and the impact of structural disorder on the critical Reynolds number is presented. Conclusions are drawn in section 4.

\section{Physical model and methodology}

\subsection{Initial boundary value problem}

The single-phase isothermal flow under consideration is that of an incompressible Newtonian fluid $\beta$ in a structure so that the solution of the is independent of the number of periodic unit cells contained in the computational domain when periodic boundary conditions are employed. Such a property is however not trivial if one is interested in unsteady flow, and this will be the object of a further analysis in section 3.1.1 as the onset of non stationary flow is under consideration in the present work.

While periodicity can be directly applied to the fluid velocity, $\mathbf{v}_{\beta}$, we shall decompose the pressure $p_{\beta}$ into its intrinsic average $\left\langle p_{\beta}\right\rangle^{\beta}$ and deviation $\tilde{p}_{\beta}$ according to $[43,44]$

$$
p_{\beta}=\left\langle p_{\beta}\right\rangle^{\beta}+\tilde{p}_{\beta}
$$

with

$$
\left\langle p_{\beta}\right\rangle^{\beta}=\frac{1}{V_{\beta}} \int_{V_{\beta}} p_{\beta} d V
$$

95

$V_{\beta}$ representing the domain occupied by the $\beta$-phase within the REV of volume $V$. With this decomposition, the gradient of average pressure can be regarded as the constant macroscopic source of the flow while $\tilde{p}_{\beta}$ is periodic.

Defining dimensionless velocity, pressure and time, $\mathbf{v}_{\beta}^{*}, p_{\beta}^{*}$ and $t_{\beta}^{*}$ from their dimensional analogs and references respectively given by $\frac{l^{2}}{\mu_{\beta}}\left|\nabla\left\langle p_{\beta}\right\rangle^{\beta}\right|, l\left|\nabla\left\langle p_{\beta}\right\rangle^{\beta}\right|$ and $\frac{\mu_{\beta}}{l}\left|\nabla\left\langle p_{\beta}\right\rangle^{\beta}\right|$ using $l$ as the reference length, 
where $l$ denotes the characteristic size of the geometrical periodic unit cell, the initial boundary value problem to be considered takes the following form

$$
\begin{gathered}
\nabla \cdot \mathbf{v}_{\beta}^{*}=0 \text { in } V_{\beta} \\
R e^{*}\left(\frac{\partial \mathbf{v}_{\beta}^{*}}{\partial t^{*}}+\mathbf{v}_{\beta}^{*} \cdot \nabla \mathbf{v}_{\beta}^{*}\right)=-\nabla \tilde{p}_{\beta}^{*}+\nabla^{2} \mathbf{v}_{\beta}^{*}-\nabla\left\langle p_{\beta}^{*}\right\rangle^{\beta}+\frac{R e^{*}}{F r} \mathbf{z} \text { in } V_{\beta} \\
\mathbf{v}_{\beta}^{*}=0 \quad \text { at } A_{\beta \sigma} \\
\mathbf{v}_{\beta}^{*}\left(\mathbf{r}^{*}+\mathbf{l}_{i}^{*}\right)=\mathbf{v}_{\beta}^{*}\left(\mathbf{r}^{*}\right) \quad i=1,2,3 \\
\tilde{p}_{\beta}^{*}\left(\mathbf{r}^{*}+\mathbf{l}_{i}^{*}\right)=\tilde{p}_{\beta}^{*}\left(\mathbf{r}^{*}\right) \quad i=1,2,3 \\
\mathbf{v}_{\beta}^{*}=0 \quad \text { in } V_{\beta} \quad \text { at } t^{*}=0 \\
\tilde{p}_{\beta}^{*}=0 \quad \text { in } V_{\beta} \text { at } t^{*}=0
\end{gathered}
$$

For the sake of simplicity, we used the same symbol for the dimensionless $\nabla(=l \nabla)$ operator. In Eq. (3c), $A_{\beta \sigma}$ represents the fluid-solid interface within $V_{\beta}$, while, in in Eqs. (3d) and (3e), the REV is characterized by the periodic lattice vectors $l_{i}, i=1,2,3$.

In the momentum equation (Eq. (3b)), Re* is the Reynolds number given by

$$
R e^{*}=\frac{\rho_{\beta} l^{3}}{\mu_{\beta}^{2}}\left|\nabla\left\langle p_{\beta}\right\rangle^{\beta}\right|
$$

In the following, we shall also use a Reynolds number based on the characteristic dimension $d$ of the solid phase already defined and related to $R e^{*}$ by

$$
R e_{d}=\rho_{\beta}\left|\left\langle\mathbf{v}_{\beta}\right\rangle\right| d / \mu_{\beta}=\left|\left\langle\mathbf{v}_{\beta}^{*}\right\rangle\right| d^{*} R e^{*}
$$

where $\langle\rangle=.\epsilon\langle.\rangle^{\beta}$ denotes the superficial average, $\epsilon=V_{\beta} / V$ being the porosity of the structure. The Reynolds number $R e_{k}$, which has the advantage of involving macroscopic quantities only, is also often used [45, 46, 23] and is obtained from $R e_{d}$ as

$$
R e_{k}=R e_{d} \frac{\sqrt{k}}{d}=R e_{d} \frac{\sqrt{k^{*}}}{d^{*}}
$$


where, for an isotropic structure, $k^{*}=k / l^{2}$ is the dimensionless form of the intrinsic permeability denoted by $k$ and $d^{*}=d / l$.

Although general, the momentum equation (3b) can be simplified by neglecting the body force term in the right hand side whenever the constraint $\frac{R e^{*}}{F r} \ll 1$ is satisfied, $F r=\frac{l^{3}\left|\nabla\left\langle p_{\beta}\right\rangle^{\beta}\right|^{2}}{\mu_{\beta}^{2} g}$ being the Froude number and $\mathbf{g}=g \mathbf{z}$ the gravity acceleration, an assumption that is adopted in the remainder of this paper.

The choice of reference pressure and length is such that the flow is driven by a unit dimensionless force

$\left(\left|\nabla\left\langle p_{\beta}^{*}\right\rangle^{\beta}\right|=1\right)$ so that the forcing is selected through the value of the Reynolds number $R e^{*}$ while flow direction is determined from the orientation of $\nabla\left\langle p_{\beta}^{*}\right\rangle^{\beta}[23]$.

The solution of the above problem to determine the critical Reynolds number value, $R e_{d c}$, corresponding to the onset of unsteady effects is carried out on $2 D$ structures for which the solid $\sigma$-phase is under the form of cylinders of square cross section. An $O S$, as represented in Fig. 1, is first considered with a porosity ranging from $15 \%$ to $96 \%$ and a macroscopic pressure gradient oriented along $\mathbf{e}_{x}$. In a second step, the impact of the flow orientation on $R e_{d c}$ is illustrated for $\epsilon=0.75$ and $\theta=\pi / 4, \theta$ being the inclination angle between the macroscopic pressure gradient and $\mathbf{e}_{x}$. Finally, a $D S$ is considered and the effect of structural disorder on $R e_{d c}$ is investigated for $\epsilon=0.75$. These two last analyses are mainly motivated by a previous investigation of inertial flow regimes in such structures that highlighted the strong dependence of these regimes upon the flow orientation and structural disorder [23].

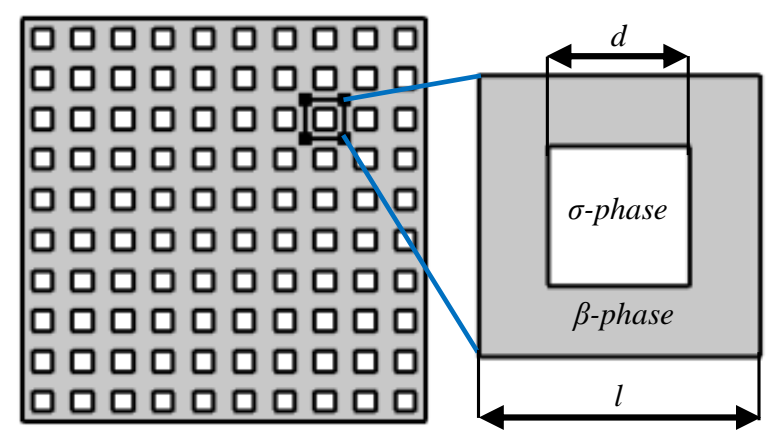

Figure 1: Large-scale structure and the periodic unit cell that corresponds to the Representative Elementary Volume for steady flow.

Rather than attempting to identify an exact numerical value of the critical Reynolds number that is extremely difficult if not impossible to achieve, the determination of $R e_{d c}$ proposed hereafter is made under the form of an interval. The lower bound of this interval corresponds to the largest value of $R e_{d}$ for which the flow remains steady remains while the upper bound corresponds to the smallest value for which non stationary flow is observed. This is achieved from a numerical solution of the initial boundary value problem (3) using a finite volume scheme as described in section 2.2 below while interval bounds are determined by 
dichotomy. The intervals are materialized on the figures throughout the article.

\subsection{Numerical method}

Simulations have been performed using the open source CFD ToolBox OpenFoam (Open Field Operation And Manipulation) based on the Finite Volume Method (FVM) of discretization [47]. The standard solver, icoFoam, which uses the PISO algorithm (Pressure Implicit Split Operator), designed for simulating transient, incompressible, laminar flow of Newtonian fluids [48], has been set to a dimensionless form by introducing the Reynolds number $R e^{*}$ and the dimensionless mean pressure gradient $\nabla\left\langle p_{\beta}^{*}\right\rangle^{\beta}$.

The Navier-Stokes equation is discretized using Euler backward discretization scheme (except for inertial term) which is second order accurate ensuring accuracy of the results [3, 34, 49, 50]. Indeed, because of incompressibility, the convective acceleration term is kept in its conservative form as $\nabla \cdot\left(\mathbf{v}_{\beta}^{*} \mathbf{v}_{\beta}^{*}\right)$ and is linearized using an explicit convective velocity, leading to a semi implcit form of the Navier-Stokes equation. For all space derivatives, the standard Gauss finite volume discretization scheme is employed with a second order central differences interpolation scheme to interpolate values from cell centers to face centers. These schemes revealed to be extremely efficient [34,3] and are optimal from the stability and accuracy points of view [50]. It must be emphazised that because of the explicit treatment of the convective term, a Courant number condition has to be satisfied in order to ensure numerical stability.

The system of linear equations for the velocity is solved using the Preconditioned Bi-Conjugate Gradient Solver (PBiCG) for asymmetric matrices and the Diagonal Incomplete Lower Upper (DILU) preconditioner. In addition, the pressure linear equation is solved using the Preconditioned Conjugate Gradient (PCG) solver for symmetric matrices and the Diagonal Incomplete Cholesky (DIC) preconditioner. Although costly in terms of memory resources because of the required matrices inversions, preconditioning operations are important in order to reduce the number of iterations performed by the linear solver to obtain a solution. A Generalised Geometric-Algebraic Multi-Grid solver (GAMG) could also be considered for the pressure equation.

Parallel computations were carried out on a 264 nodes cluster of 12 Intel@Xeon $囚 \times 5675(3,06 \mathrm{GHz})$ cores each.

\subsection{Convergence criterion and mesh sensitivity}

The choice of a consistent dimensionless time step $\Delta t^{*}$ has been made depending on grid-block size and velocity magnitude. More precisely, a $C F L$ condition must be verified over the whole mesh for the different structures under consideration. It is expressed as

$$
C o=\frac{\Delta t^{*}\left|\mathbf{v}_{\beta}^{*}\right|}{\Delta s^{*}}<C o_{\max }
$$


where $C o$ represents the Courant number on each grid-block, $\Delta s^{*}$ being the dimensionless grid-block size in the direction of the velocity. In this work, $C o_{\max }=0.5$, which means that a fluid particle will not move over more than half a grid-block over a time step as proposed in [51]. Moreover, the choice of a consistent time step depends also on the oscillation frequency (for unsteady flow). It has been demonstrated that 500 time steps per cycle are generally needed $[52,53,54]$ and we have also taken this latter condition under consideration.

The steady/unsteady character of the flow is determined from the convergence criterion set for the time dependent problem in Eqs. (3). Starting initially from rest, the flow enters a transient period until it reaches stationarity if the Reynolds number is subcritical. This transient period grows longer as $R e^{*}$ increases.

The system of partial differential equations is solved with a segregated approach $[55,56]$ yielding three linear systems for $v_{\beta x}^{*}, v_{\beta y}^{*}$ and $\tilde{p}_{\beta}^{*}$. Given the solution of these systems at time $t^{*(n)}$, which is injected as an initial guess in the linear systems at time $t^{*(n)}+\Delta t^{*}$, residuals are computed as the $L_{1}-$ norm of the normalized difference between the right and left hand sides of each linear system. If these residuals are all smaller than the user-prescribed tolerances, the flow is considered as steady and the simulation ends. If not, successive iterations are performed by the linear solver until the residuals are less than the tolerances providing the solution at time $t^{*(n)}+\Delta t^{*}$.

Tolerances for the linear system solvers (PBiCG for $v_{\beta x}^{*}$ and $v_{\beta y}^{*}$ and PCG for $\tilde{p}_{\beta}^{*}$ ) were respectively set to $10^{-10}$ and $10^{-7}$. A special attention was dedicated to the choice of these values so as to ensure an accurate estimation of $R e_{d c}$. An illustrative example of the dependence of $R e_{d c}$ on the tolerance for PBiCG solver is provided in Fig. 2 for the most restrictive porosity $(\epsilon=96 \%)$ which clearly justifies the $10^{-10}$ value. Setting the tolerance for the PBiCG solver to $10^{-10}$, the relative error on the computed $R e_{d c}$, using $10^{-7}$ and then $10^{-10}$ as the tolerance for the PCG solver, is found to be less than 1\%. Similar tests carried out on larger REVs as employed in section 3.1.1 yield similar conclusions. These values represent significantly more severe tolerances than the one classically used in works based on the same approach and reported earlier in which a tolerance of $10^{-5}$ on both the velocity components and pressure residuals was considered to characterize flow steadiness [51]. It should also be noted that stationarity has to be verified over a sufficiently large number of time steps in order to ensure that the flow field is fully developed and that no bifurcation is to occur. In the present analysis, in addition to the condition on the residuals, the bifurcation towards unsteadiness is checked from the symmetry-breaking of the flow, i.e. when the spatial average of the velocity component perpendicular to $\nabla\left\langle p_{\beta}^{*}\right\rangle^{\beta}$ becomes non-zero [57]. This could also be performed using a spectral analysis of this velocity component with an FFT (Fast Fourier Transform), an example of which is reported in section 3.1.3. It must be emphasized that Fast Fourier Transforms have to be carried out over sufficiently large data samples in order to capture very low frequency peaks [58]. Our dimensionless simulation times range from $\sim 6.3 \times 10^{4}\left(\sim 2.5 \times 10^{6}\right.$ time steps $)$ for $\epsilon=96 \%$ to $\sim 2.1 \times 10^{6}\left(\sim 83.4 \times 10^{6}\right.$ time steps $)$ for $\epsilon=15.36 \%$. 


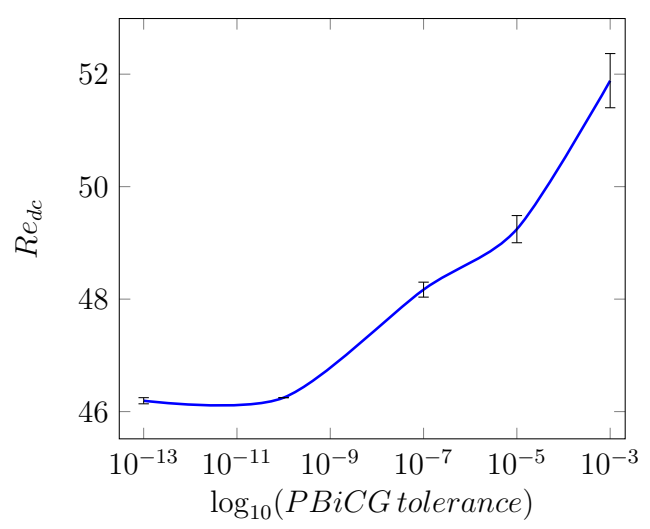

Figure 2: Critical Reynolds number $R e_{d c}$ versus velocity tolerance of the PBiCG solver. Vertical bars materialize the upper and lower bounds of $\operatorname{Re}_{d}$ at which unsteady and steady flows are respectively observed. Pressure deviation tolerance is set to $10^{-7}$. OS (see Fig. 1). $\nabla\left\langle p_{\beta}^{*}\right\rangle^{\beta}=\mathbf{e}_{x} \cdot \epsilon=96 \%$.

Structured hexahedral meshes (cubic grid-blocks) and extruded triangles (triangular prisms) were employed for $O S$ and $D S$ respectively and were generated with the open source mesher Salome. It is well known that to avoid spurious oscillations associated with the central difference scheme for the nonlinear terms, fine meshes should be adopted to perform accurate simulations [50]. Sensitivity to grid-block size was 
that mesh convergence is correctly captured with the selected grid-block sizes.

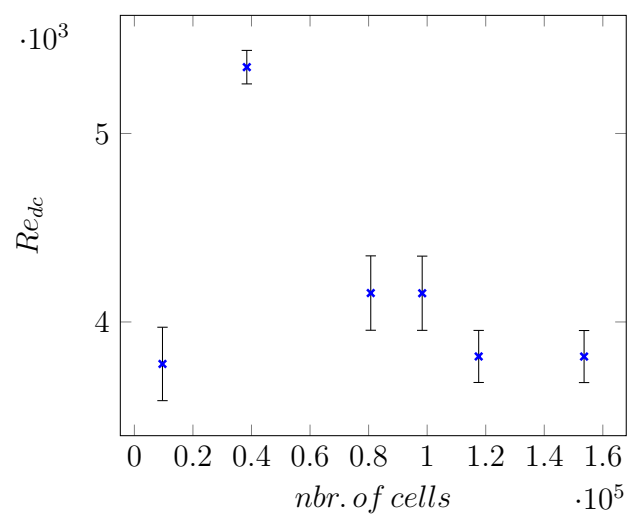

a) $\epsilon=15.36 \%$

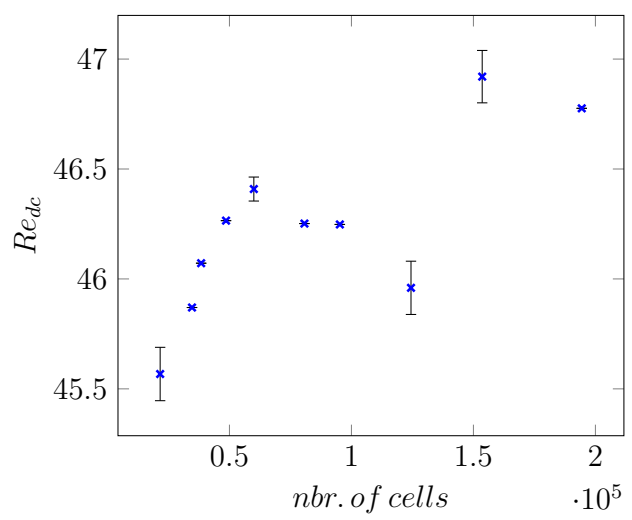

b) $\epsilon=96 \%$

Figure 3: Critical Reynolds number $R e_{d c}$ versus the number of grid-blocks. Vertical bars materialize the upper and lower bounds of $R e_{d}$ at which unsteady and steady flows are respectively observed. $O S . \nabla\left\langle p_{\beta}^{*}\right\rangle^{\beta}=\mathbf{e}_{x}$.

For $D S$, due to the size of computational domains (see Section 3.2), a coarser mesh was employed increasing the grid-block size by a factor ranging from 5 (for $\epsilon=36 \%$ ) to 10 (for $\epsilon=75 \%$ ).

A dimensionless time step $\Delta t^{*}=0.025$, satisfying the condition (7), suitable to achieve temporal accuracy and numerical stability has been adopted for all our simulations.

\subsection{Validation}

Due to the lack of bibliography treating the first Hopf bifurcation on periodic structures, the methodology has been validated on the classical case of flow past a cylinder of square cross section (Fig. 4). 


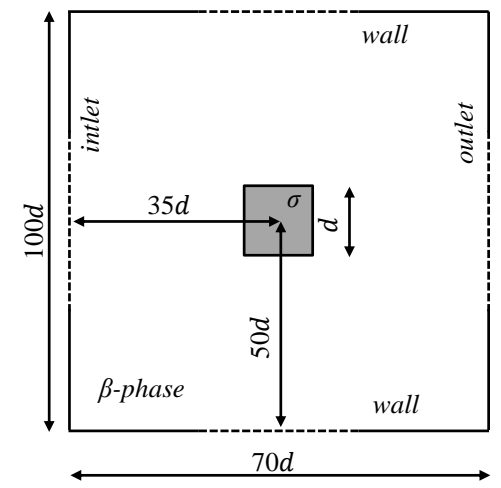

(a) Computational domain

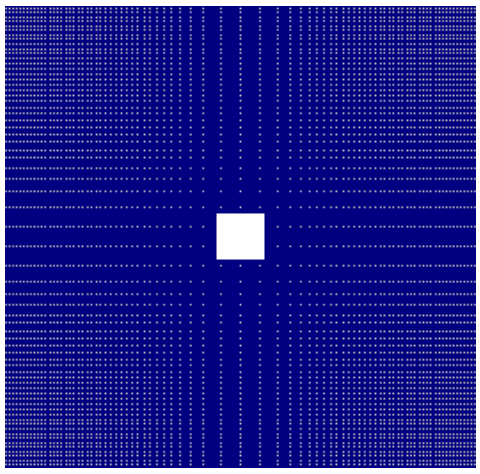

(b) close-up view of the cylinder

Figure 4: Computational domain for flow simulation past a cylinder of square cross section and detail of the mesh close to the cylinder.

The corresponding dimensionless single-phase isothermal flow problem of an incompressible Newtonian fluid $\beta$ is given by (we omit the ${ }^{*}$ for the dimensionless $\nabla$ operator)

$$
\begin{gathered}
\frac{\partial \mathbf{v}_{\beta}^{*}}{\partial t^{*}}+\mathbf{v}_{\beta}^{*} \cdot \nabla \mathbf{v}_{\beta}^{*}=-\nabla p_{\beta}^{*}+\frac{1}{R e} \nabla^{2} \mathbf{v}_{\beta}^{*}+\frac{\mathbf{z}}{F r} \\
\nabla \cdot \mathbf{v}_{\beta}^{*}=0
\end{gathered}
$$

The associated boundary conditions are

$$
\begin{gathered}
\mathbf{v}_{\beta}^{*}=0 \quad \text { at } A_{\beta \sigma} \text { and wall } \\
\mathbf{v}_{\beta}^{*}=\mathbf{e}_{x} \text { at inlet } \\
p_{\beta}^{*}=0 \text { at outlet }
\end{gathered}
$$

Starting with the fluid at rest, initial conditions are given by

$$
\left\{\begin{array}{l}
\mathbf{v}_{\beta}^{*}=0 \\
p_{\beta}^{*}=0
\end{array} \quad \text { at } t^{*}=0\right.
$$

The dimensionless velocity $\mathbf{v}_{\beta}^{*}$, pressure $p_{\beta}^{*}$ and time $t^{*}$ are respectively defined from the modulus of the (uniform) inlet velocity, $v_{r e f}, \rho_{\beta} v_{r e f}^{2}$, and $d / v_{r e f}$ while all coordinates are made dimensionless by $d$, the side length of the square cross section of the cylinder (cf. Fig. 4). 
The Reynolds and Froude numbers in Eq. (8) are respectively given by

$$
R e=\frac{\rho_{\beta} v_{r e f} d}{\mu_{\beta}} \quad F r=\frac{v_{r e f}^{2}}{g d}
$$

Again, we assume $F r \gg 1$ in the context of negligible gravity effects.

To reproduce the flow in an infinite domain, the inlet uniform Dirichlet velocity (Eq. (11)) and outlet zero pressure boundary conditions (Eq. (12)) were respectively imposed at a distance $35 d$ upstream and downstream the cylinder center. Similarly, upper and lower walls were positioned at a distance equal to 50 above and below the cylinder center.

The domain (see Fig. 4) was discretized using a structured grid of hexahedral blocks (with a single 240 element in the cylinder axis direction) made of 423300 grid blocks with a refinement around the square cylinder as shown in Fig. 4. Each side of the square cylinder is in contact with 25 grid blocks and the grid-block size is linearly decreased by a factor of 8 from the boundaries to the fluid-solid interfaces. 


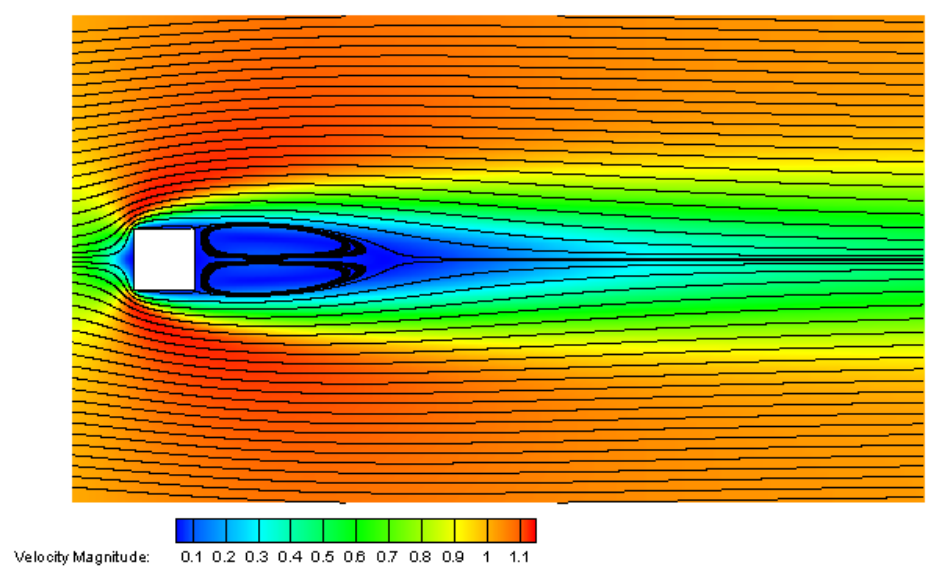

(a) Laminar steady flow regime. $R e=45 . t^{*}=1400$.

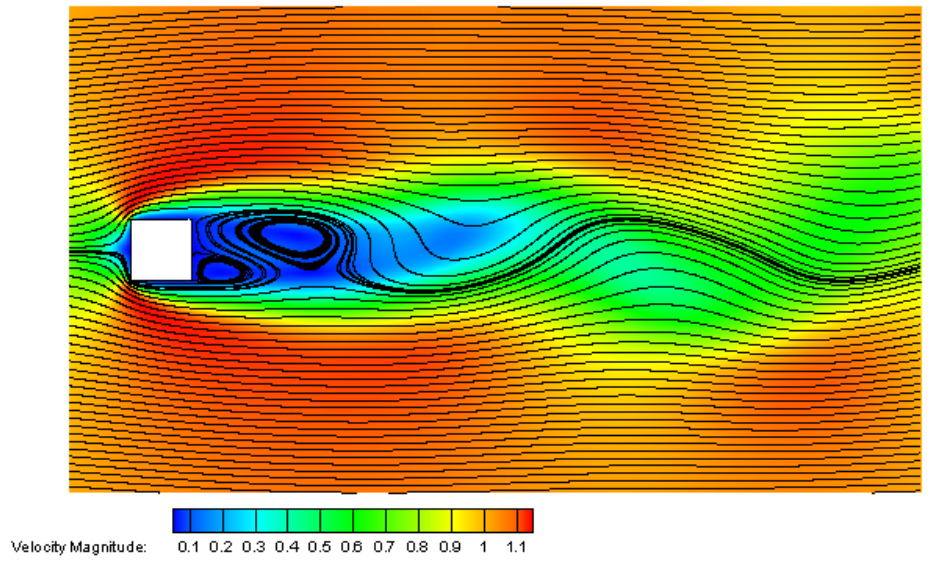

(b) Laminar unsteady flow regime. $R e=50 . t^{*}=1000$.

Figure 5: Streamlines and velocity magnitude color map for flow past a unique cylinder of square cross section.

The critical Reynolds number $R e_{c}$ is defined based on the fixed convergence criterion mentioned in section 2.3. Results are supported by symmetry-breaking that yields an instantaneous non-zero space average velocity along $\mathbf{e}_{y}$ as can be inferred from Fig. 5b. Steady flow is characterized by symmetric streamlines (see Fig. 5a) while laminar unsteady flow corresponding to the Hopf bifurcation is characterized by oscillating streamlines and the emergence of a von Kármán vortex street. The critical Reynolds number leading to the Hopf bifurcation and the apparition of vortex shedding phenomenon obtained from our simulations is $R e_{c}=46 \pm 1$ in agreement with reported values in many references. Indeed, exactly the same value was obtained in a numerical work by Lankadasu et al. [59] while Yoon et al. obtained $R e_{c}=45$ from their simulation [35]. Experimentally, Sohankar et al. [60] identified $R e_{c}=47 \pm 2$.

To confirm the accuracy of the adopted methodology, the critical Reynolds number was also computed for an ordered structure made of a square pattern of parallel cylinders of circular cross section with $\epsilon=80 \%$, using 
a geometrical periodic unit cell and periodic boundary conditions on velocity and pressure deviation (Fig. 6). For this structure, the critical Reynolds number is found to be $R e_{d c}=118,45 \pm 4,92\left(R e_{c}^{*}=16250 \pm 750\right)$, which is in agreement with a value obtained by Koch and Ladd [39], $R e_{d c} \approx 122,9 \pm 2,4$. A relatively different value was reported earlier, $R e_{d c} \approx 144,25 \pm 5,95$ [51]. However, unlike the former work, the later value was obtained without a mesh sensitivity analysis which may explain why the value of $R e_{d c}$ is larger.

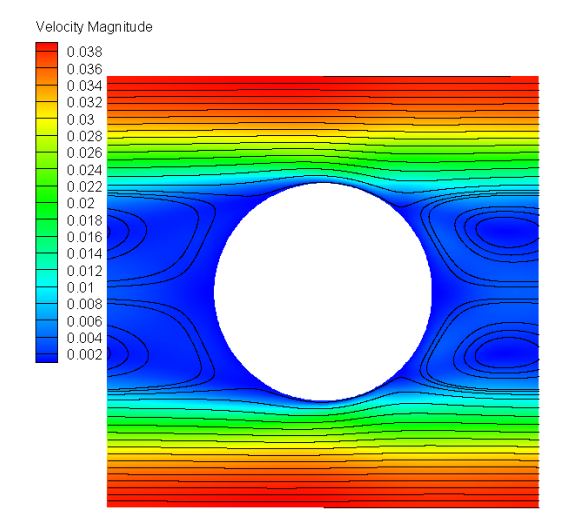

(a) Steady flow. $R e_{d}=141.91\left(R e^{*}=\right.$ $15500) \cdot t^{*}=15000$.

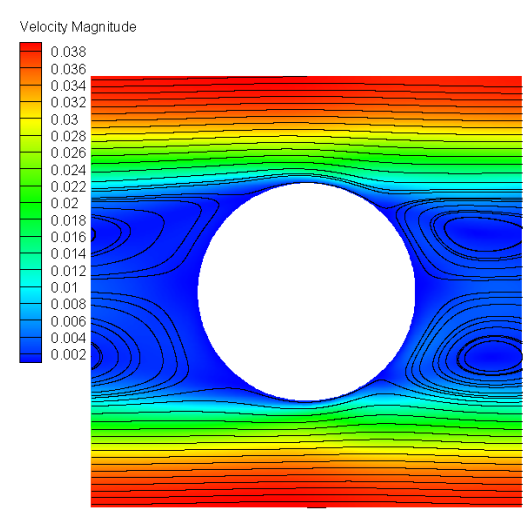

(b) Unsteady flow. $R e_{d}=154.20\left(R e^{*}=\right.$ 17000). $t^{*}=14000$.

Figure 6: Streamlines and velocity magnitude color maps. Ordered structure made of a square pattern of parallel cylinders of circular cross section. The REV is taken as the geometrical periodic unit cell. $\epsilon=80 \% \cdot \nabla\left\langle p_{\beta}^{*}\right\rangle^{\beta}=\mathbf{e}_{x}$.

\section{Results and discussion}

\subsection{Ordered structure}

\subsubsection{Dependence of $R e_{d c}$ on the $R E V$ size}

As stated before, the REV for the permanent version of the flow problem (Eqs. (3)) in the case of a geometrically periodic structure like the $O S$ of figure 1 corresponds to the geometrical periodic unit cell of size $l$. This periodic unit cell has been considered as a REV using periodic boundary conditions on $\mathbf{v}_{\beta}^{*}$ and $\tilde{p}_{\beta}^{*}$ in many reported works, both in steady and unsteady flow conditions [51, 61, 39, 62]. The implicit underlying hypothesis in the latter case is that periodic boundary conditions at scale $l$ do not have any effect on the flow structuring and hence on the critical Reynolds number.

In order to investigate the impact of the periodic boundary conditions on the onset of the Hopf bifurcation, the critical Reynolds number $R e_{d c}$ was evaluated for the $O S$ and a porosity $\epsilon=75 \%$ over REVs of different sizes. Computations were first performed over a geometrical periodic unit cell (see Fig. 7) and the resulting value of $R e_{d c}$ was then compared to that obtained while considering a REV composed of $3 \times 3$ geometrical unit cells (see Fig. 8). 


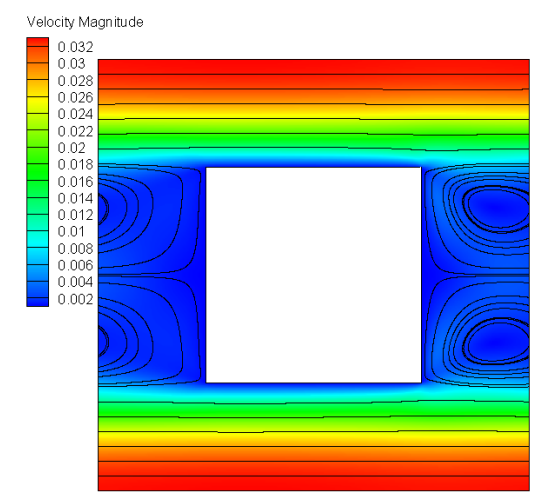

(a) Limit of the steady flow. $R e_{d}=162.05$

$\left(R e_{d c}=163.92 \pm 1.87\right)$.

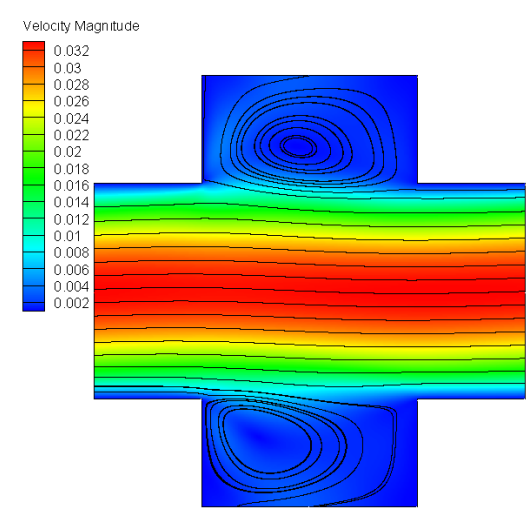

(c) Unsteady flow. $R e_{d}=165.79\left(R e_{d c}=\right.$ $163.91 \pm 1.86)$.

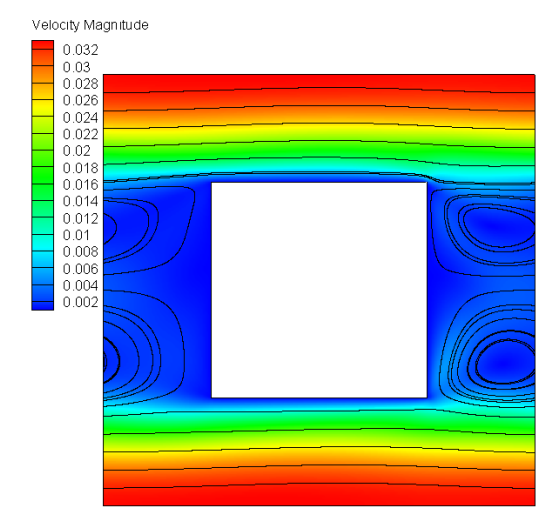

(b) Unsteady flow. $R e_{d}=165.73\left(R e_{d c}=\right.$ $163.92 \pm 1.87)$.

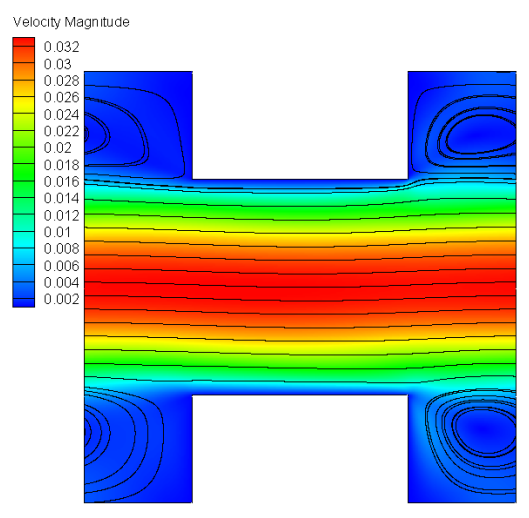

(d) Unsteady flow. $R e_{d}=165.73\left(R e_{d c}=\right.$ $163.91 \pm 1.85)$.

Figure 7: Streamlines and velocity magnitude color maps. For unsteady flows, snapshots are at $t^{*}=43000$ for the same $\nabla\left\langle p_{\beta}\right\rangle^{\beta}$ (i.e. the same $\left.R e^{*}=29000\right)$. OS. The REV is taken as the geometrical periodic unit cell and shifted along the axes of the cylinders lattice. $\epsilon=75 \% . \nabla\left\langle p_{\beta}^{*}\right\rangle^{\beta}=\mathbf{e}_{x} . R e_{c}^{*}=28500 \pm 500$.

Simulations on the unit cell of Fig. 7 with periodic boundary conditions on $\mathbf{v}_{\beta}^{*}$ and $\tilde{p}_{\beta}^{*}$ lead to a critical Reynolds number $R e_{d c}=163.92 \pm 1.87\left(R e_{c}^{*}=28500 \pm 500\right)$. The steady flow (see Fig. 7a) is characterized by straight streamlines along the average pressure gradient direction $\nabla\left\langle p_{\beta}^{*}\right\rangle^{\beta}=\mathbf{e}_{x}$ near the the top and bottom planes of symmetry of the unit cell and two symmetric vortices that fill the gap between successive cylinders. The onset of the Hopf bifurcation (Fig. 7b) is characterized by oscillating vortices and streamlines.

First, it must be made clear that the value of $R e_{d c}$ remains unmodified, irrespective of the $x$ and $y$ positions of the periodic unit cell relative to the cylinders lattice. This was checked for the three unit cells depicted in figures $7 \mathrm{~b}, 7 \mathrm{c}$ and $7 \mathrm{~d}$ which show, however, that the structure of the unsteady flow at a given $t^{*}$ slightly differs from one unit cell to another. 


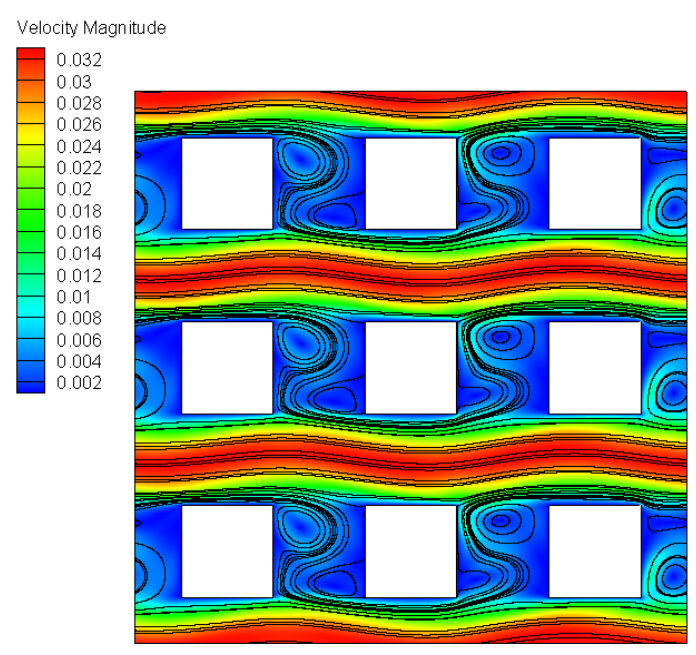

Figure 8: Instantaneous streamlines and velocity magnitude color map. OS. REV composed of $3 \times 3$ geometrical unit cells. $\epsilon=75 \%, \nabla\left\langle p_{\beta}^{*}\right\rangle^{\beta}=\mathbf{e}_{x}$. Unsteady flow, $R e_{d}=137.08\left(R e^{*}=25000\right), t^{*}=6000$.

Second, for the same porosity value $(\epsilon=75 \%)$, the critical Reynolds number $R e_{d c}$ determined on a REV composed of $3 \times 3$ geometrical periodic unit cells of the $O S$ is found to be $R_{d c}=100.31 \pm 2.28$ $\left(R e_{c}^{*}=17100 \pm 400\right)$, a value significantly different from the former, yielding a relative error of $\sim 63 \%$ that of streamlines and velocity magnitude color maps are represented at the same $t^{*}$ and four different REVs. 
For $\epsilon=96 \%$, for which the REV size effect is expected to be very significant, a relative error of less than $5 \%$ was found on $R e_{d c}$ between REVs made of $5 \times 1\left(R e_{d c}=12.82 \pm 3.94, R e_{c}^{*}=1125 \pm 375\right)$ and $3 \times 1$ the REV allowing the determination of $R e_{d c}$ over the whole range of $\epsilon$ as reported below.

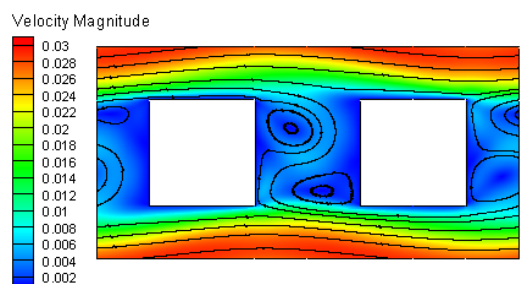

(a) $R e_{d}=121.24 .2 \times 1$ geometrical unit cells $\left(R e_{d c}=96.41 \pm 2.37, R e_{c}^{*}=16875 \pm\right.$ $875)$.

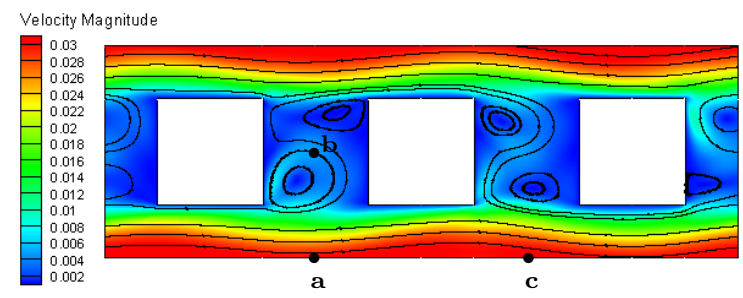

(b) $R e_{d}=129.46 .3 \times 1$ geometrical unit cells $\left(R e_{d c}=\right.$ $\left.100.82 \pm 2.5, R e_{c}^{*}=17250 \pm 500\right)$. The spectral analysis of section 3.1 .3 is performed at the reference points (a), (b) and (c).

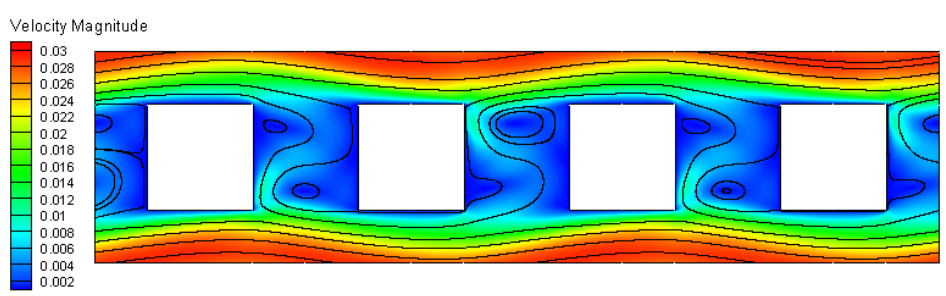

(c) $R e_{d}=120.92 .4 \times 1$ geometrical unit cells $\left(R e_{d c}=96.38 \pm 2.35, R e_{c}^{*}=\right.$ $16875 \pm 875)$.

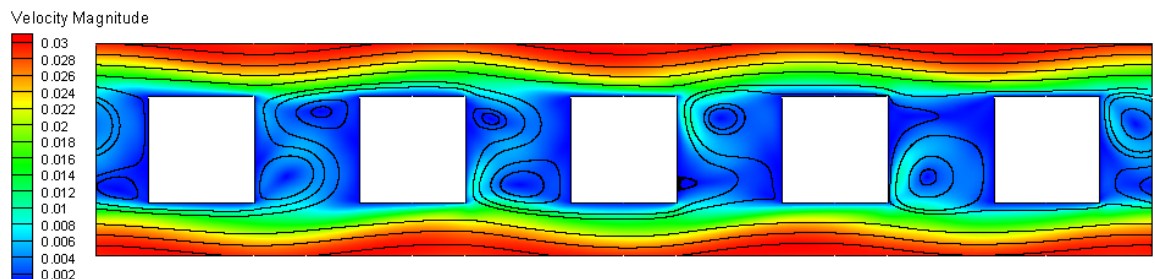

(d) $R e_{d}=125.74 .5 \times 1$ geometrical unit cells $\left(R e_{d c}=96.17 \pm 2.99, R e_{c}^{*}=16800 \pm 950\right)$.

Figure 9: Instantaneous streamlines and velocity magnitude color maps at the same $t^{*}=9000$ and for the same $\nabla\left\langle p_{\beta}\right\rangle^{\beta}($ i.e. the same $\left.R e^{*}=25000\right)$. OS. REVs of different sizes are considered. $\epsilon=75 \% . \nabla\left\langle p_{\beta}^{*}\right\rangle^{\beta}=\mathbf{e}_{x}$.

\subsubsection{Dependence of $\operatorname{Re}_{d c}$ on $\epsilon$}

In Fig. 10, we have reported our results on $R e_{d c}$ versus $\epsilon$ in the range $15 \% \leq \epsilon \leq 96 \%$ for the $O S$ along with those obtained with a REV corresponding to the geometrical periodic unit cell for $\epsilon \geq 45 \%$. Below this value, the Reynolds number characterizing the Hopf bifurcation is not significantly modified by the choice of the REV size, while, above this value, the overestimation of $R e_{d c}$ increases strongly with $\epsilon$ when the REV corresponds to the periodic unit cell, $R e_{d c}$ being roughly four times larger (46.78 instead of 12.0) with this 
latter choice for $\epsilon=96 \%$.

Two extreme situations may be considered for $\epsilon$ exceedingly close to the limiting values 1 and 0 . In the

315 former case, one could call upon an analogy with flow around a unique cylinder in an infinite domain as envisaged in section 2.4 for which $R e_{c}=46$. However, periodic conditions on both velocity and pressure deviation on the REV boundaries lead to a flow structure significantly different. In fact, periodicity implies that the flow upstream each cylinder is non uniform as it is conditioned by the preceding obstacle, introducing a perturbation. Consequently, the value of $R e_{d c}$ is expected to be significantly smaller and this is indicated by our results yielding $R e_{d c}=11.97\left(R e_{c}^{*}=1075 \pm 25\right)$ for the $O S$ and $\epsilon=96 \%$.

When $\epsilon$ is extremely small, an analogy with the flow between plane parallel plates is appealing. The critical Reynolds number corresponding to the first Hopf bifurcation is known to be $R e_{e c} \approx 5772.22$ in this plane Poiseuille flow configuration [63], $R e_{e c}$ being estimated with half the plates spacing and the velocity magnitude on the centerline of the channel. The critical Reynolds number obtained on the $O S$ for the lowest porosity investigated in this work $(\epsilon=15.36 \%$, see Fig. 10$)$ is $R e_{d c}=3816.99 \pm 138.14$ $\left(R e_{c}^{*}=9.65 \times 10^{7} \pm 3.5 \times 10^{6}\right)$, which, expressed in terms of $R e_{e c}$, yields $R e_{e c}=3104.41 \pm 112.41$. This significantly smaller value is not surprising since the presence of the channels in the direction perpendicular to the average pressure gradient for the $O S$ are singularities in the region of which eddies are generated, yielding perturbations that trigger the bifurcation at a Reynolds number smaller than that expected in the case of continuous plates.

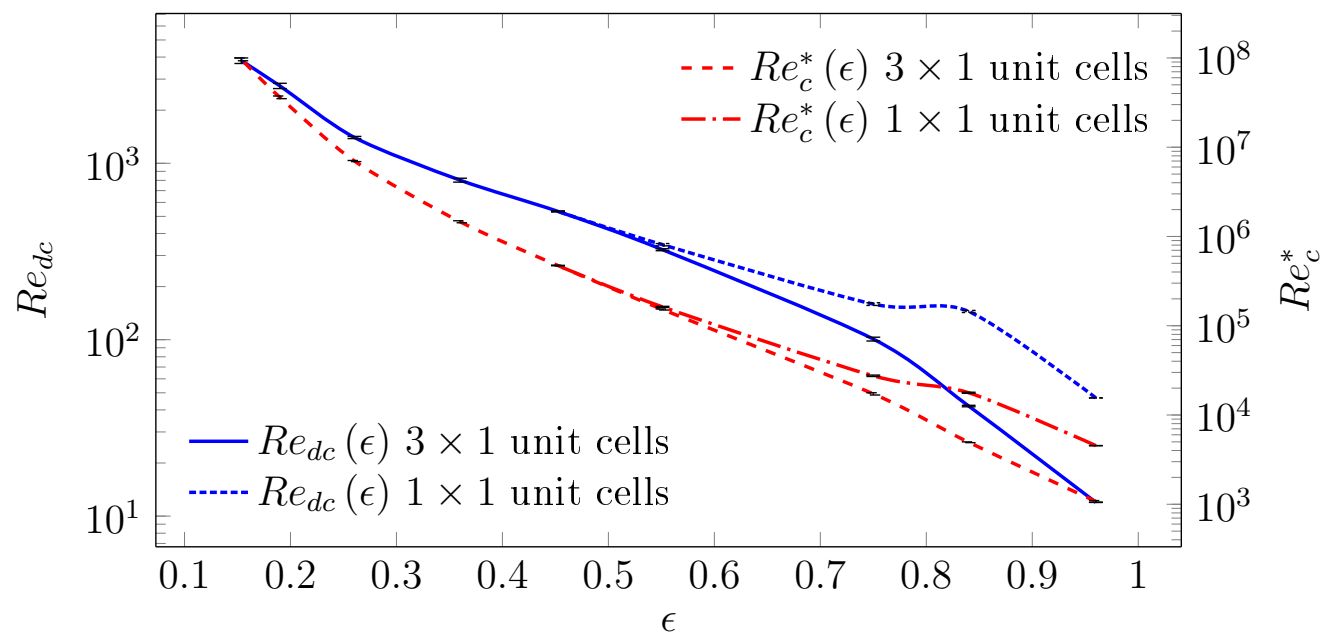

Figure 10: Dependence of the critical Reynolds number, $R e_{d c}$ (or $R e_{c}^{*}$ ), on $\epsilon$. Vertical bars (unobservable here) materialize the upper and lower bounds of $\operatorname{Re}_{d}$ (or $R e^{*}$ ) at which unsteady and steady flows are respectively observed. $O S . \nabla\left\langle p_{\beta}^{*}\right\rangle^{\beta}=\mathbf{e}_{x}$.

In the porosity range under investigation $15.36 \% \leq \epsilon \leq 96 \%, R e_{d c}$ monotonically decreases with $\epsilon$ (see Figs. 10 and 11). This behavior is in agreement with recent experimental results on floor water inrush [40]. 
Physically, this can be explained by the fact that, for small porosities, the flow is strongly oriented and constrained within the horizontal channels while the kinetic energy carried in the eddies in the gaps between two successive cylinders which may destabilize the flow, is weak compared to the case of larger porosities.

The graph of figure 10 suggests that sigmoidal fits can be proposed, given by

$$
R e_{d c}=a_{0}\left(\frac{1-\epsilon}{b_{0}+\epsilon}\right)^{c_{0}}
$$

with $a_{0}=383.75, b_{0}=-0.02, c_{0}=1.23$ for $0.1536 \lesssim \epsilon \lesssim 0.75$ and $a_{0}=40.17, b_{0}=-0.69, c_{0}=0.636$ for $0.75 \lesssim \epsilon \lesssim 0.96$, yielding Pearson correlation coefficients $[64,65] R^{2}=0.9987$ and $R^{2}=0.9999$ respectively. Our intrinsic permeability results, that may be used to compute $R e_{k}$ (see Eq. (6)), yield correlations with $\epsilon$ given by

$$
k^{*}=a_{1} \frac{\epsilon^{b_{1}}}{(1-\epsilon)^{c_{1}}}
$$

with $a_{1}=0.0123, b_{1}=3.07, c_{1}=0.707$ for $0.1536 \lesssim \epsilon \lesssim 0.75$ and $a_{1}=0.023, b_{1}=3.82, c_{1}=0.39$ for $0.75 \lesssim \epsilon \lesssim 0.96$, the correlation coefficient being $R^{2}=0.9999$

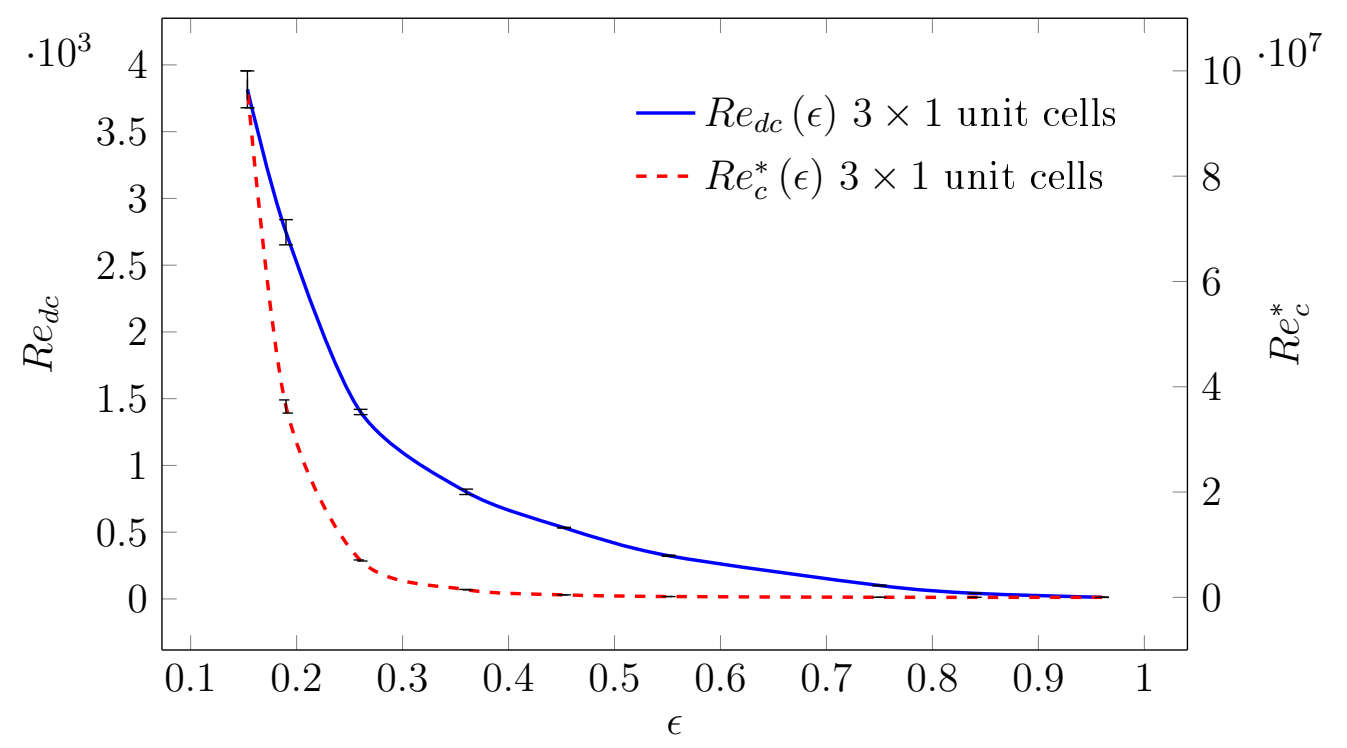

Figure 11: Dependence of the critical Reynolds number, $R e_{d c}$ (or $R e_{c}^{*}$ ), on $\epsilon$ and sigmoidal fit curves (see text). Vertical bars materialize the upper and lower bounds of $R_{d}$ (or $R e^{*}$ ) at which unsteady and steady flows are respectively observed. OS. $\nabla\left\langle p_{\beta}^{*}\right\rangle^{\beta}=\mathbf{e}_{x}$. REV made of $3 \times 1$ geometrical unit cells.

While increasing the Reynolds number from the creeping regime up to unsteadiness, the flow structure resulting from $\nabla\left\langle p_{\beta}^{*}\right\rangle^{\beta}=\mathbf{e}_{x}$ on the $O S$ is characterized by two main features, as depicted in Fig. 12a-d for $\epsilon=75 \%$. First, streamlines in the horizontal gaps between the cylinders become less tortuous and, second, eddies in the vertical gap, which remain symmetric, are simultaneously growing until they occupy the whole space between two successive cylinders. At this stage that just precedes the Hopf bifurcation (see Fig. 12d), 
the flow along $\nabla\left\langle p_{\beta}^{*}\right\rangle^{\beta}$ within the horizontal gaps resembles that between plane parallel plates as observed elsewhere [61].

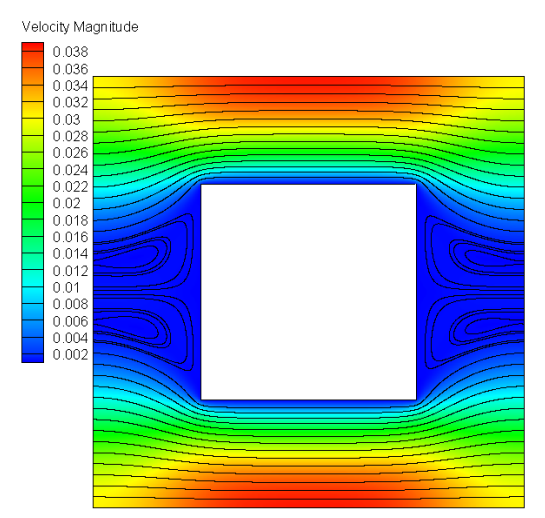

(a) Steady creeping flow. $R e_{d}=0.33$ $\left(R e^{*}=50\right)$.

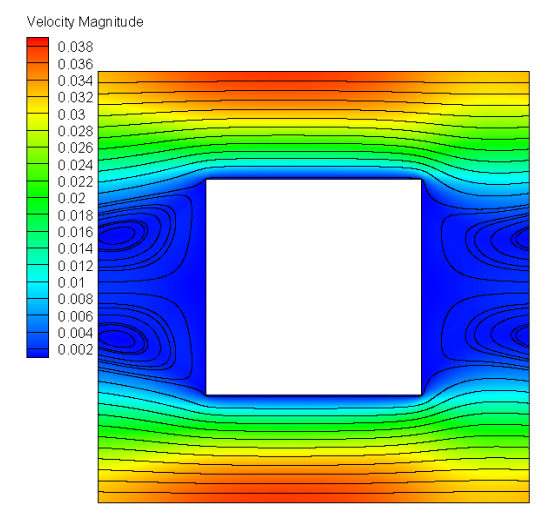

(c) Steady strongly inertial flow. $R e_{d}=$ $9.53\left(R e^{*}=1500\right)$.

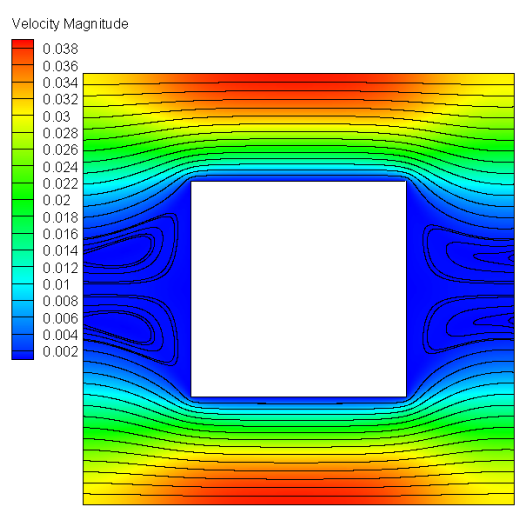

(b) Steady weakly inertial flow. $R e_{d}=1.82$ $\left(R e^{*}=280\right)$.

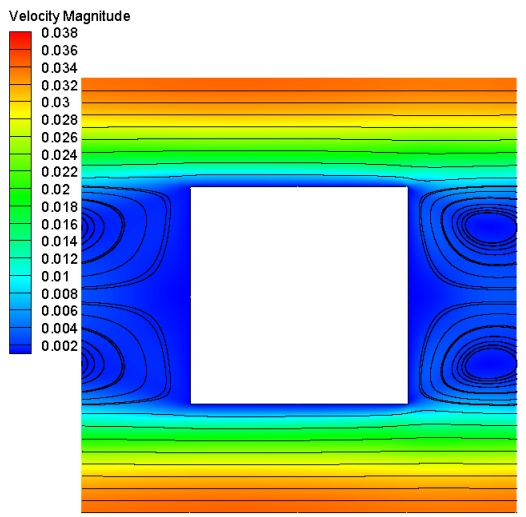

(d) Limit of the steady flow. $R e_{d}=98.32$ $\left(R e^{*}=17000\right)$.

Figure 12: Streamlines and velocity magnitude color maps. OS. $\epsilon=75 \% . \nabla\left\langle p_{\beta}^{*}\right\rangle^{\beta}=\mathbf{e}_{x}$.

This kind of behavior is observed over a wide range of porosity values, with, in particular, a pair of developing symmetric eddies (just as for a unique cylinder) in the vertical gaps. However, for small enough porosities, this pattern is modified as this is clearly evidenced on Fig. 13 where six vortices are obtained for $\epsilon=15.36 \%$ (Fig. 13a) leaving a quasi-dead zone in the center of the vertical gap. For $\epsilon=36 \%$, four vortices can be observed (Fig. 13b) occupying the whole vertical gap, in contrast with the case for $\epsilon=96 \%$ where the two symmetric vortices do not have a horizontal extent large enough to occupy the whole region between successive cylinders, even at the Hopf bifurcation threshold (Fig. 13c). Clearly, more work, that is however beyond the scope of the present investigation, is required to correlate the observations on the 
flow structure and its evolution with the Reynolds number to the different regimes (in particular the weak and strong inertia regimes and the transition between the two) highlighted in previous works in the same geometrical configuration [23].

In all situations of the $O S$ with $\nabla\left\langle p_{\beta}^{*}\right\rangle^{\beta}=\mathbf{e}_{x}$, when the critical Reynolds number $R e_{d c}$ is reached, unsteadiness is first observed on the eddies which become asymmetric and start oscillating vertically with varying periods and amplitudes as pointed out elsewhere [66, 51], propagating a perturbation on the entire flow over the whole structure (Fig. 8). This is the object of a more thorough analysis in the next section 3.1.3.

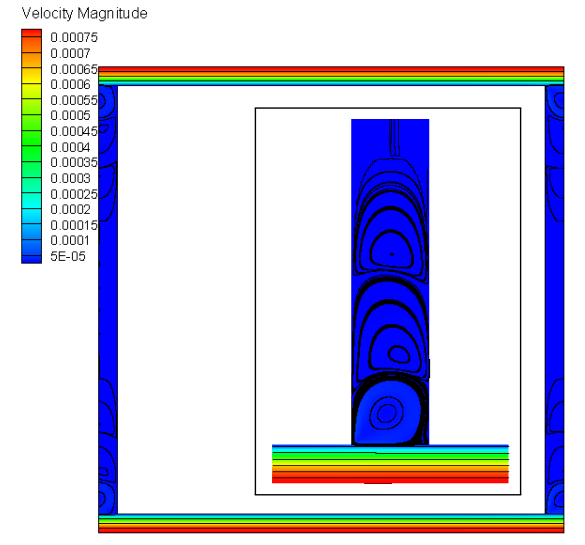

(a) $\epsilon=15.36 \%$. REV composed of $1 \times 1$ geometrical unit cell. $R e_{d}=3678.85\left(R e^{*}=\right.$ $\left.9.3 \times 10^{7}\right)$. Inset: Close-up view of the lowerhalf vertical gap.

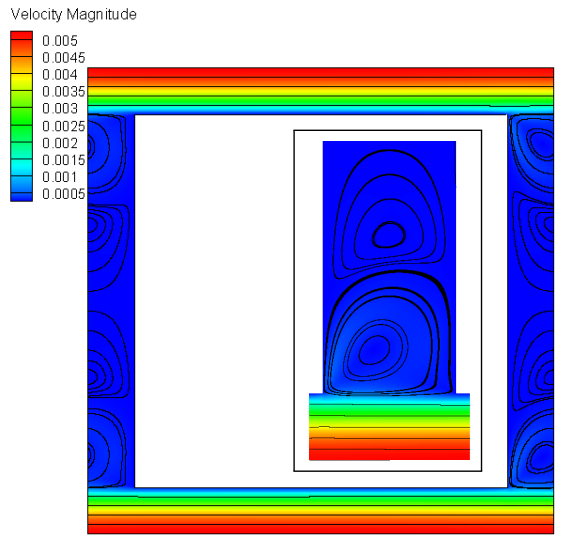

(b) $\epsilon=36 \%$. REV composed of $1 \times 1$ geometrical unit cell. $R e_{d}=782\left(R e^{*}=1.425 \times 10^{6}\right)$. Inset: Close-up view of the lower-half vertical gap.

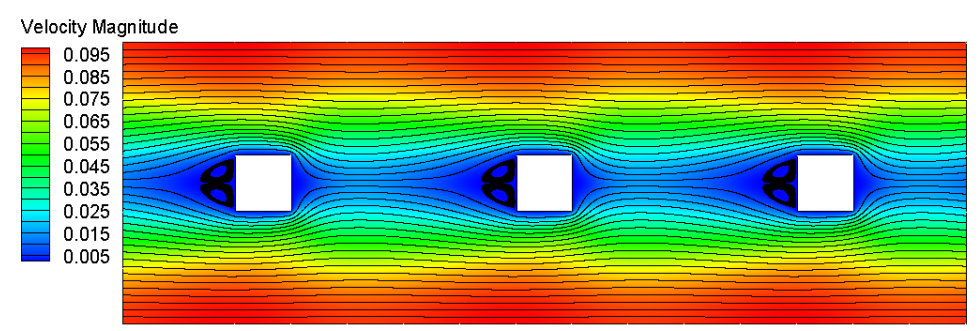

(c) $\epsilon=96 \%$. REV composed of $3 \times 1$ geometrical unit cells. $R e_{d}=11.96$ $\left(R e^{*}=1050\right)$.

Figure 13: Streamlines and velocity magnitude color maps. OS. $\nabla\left\langle p_{\beta}^{*}\right\rangle^{\beta}=\mathbf{e}_{x}$. Limit of steady flow.

\subsubsection{Spectral analysis on $v_{\beta y}^{*}$}

Time evolution of the dimensionless transverse velocity $v_{\beta y}^{*}$ in the $O S$ made of $3 \times 1$ geometrical unit cells, for $\epsilon=75 \%$ and $\nabla\left\langle p_{\beta}^{*}\right\rangle^{\beta}=\mathbf{e}_{x}$ is now analyzed for different Reynolds numbers at three different points 

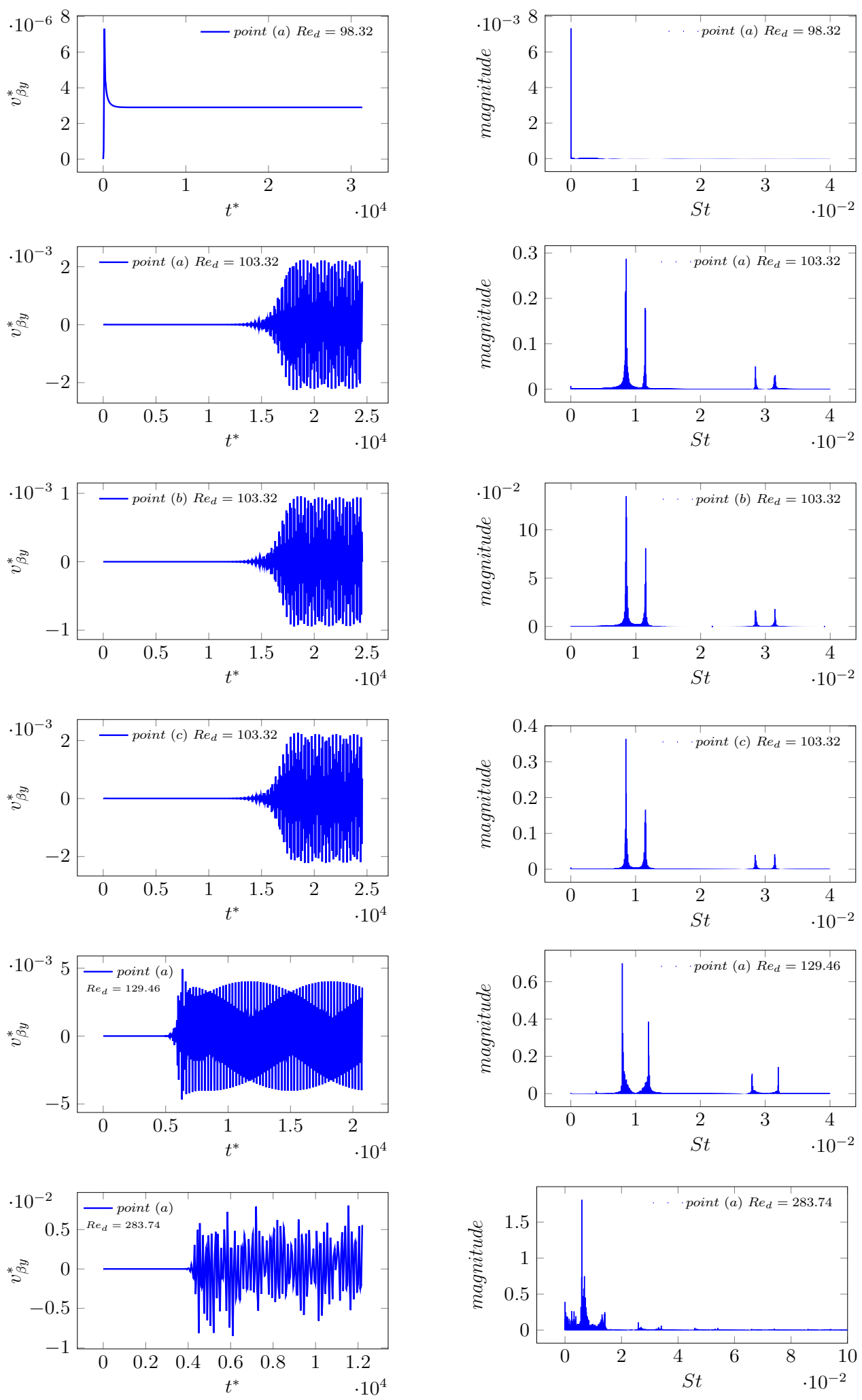

Figure 14: Dimensionless $y$-component of the fluid velocity versus dimensionless time at reference points (a), (b) and (c) as reported in Fig. 9b and corresponding spectra versus the Strouhal number St. OS. REV composed of $3 \times 1$ geometrical unit cells. $\epsilon=75 \% . \nabla\left\langle p_{\beta}^{*}\right\rangle^{\beta}=\mathbf{e}_{x}$. 
(see Fig. 9b) located at the center of the channel parallel to $\nabla\left\langle p_{\beta}^{*}\right\rangle^{\beta}$, (point (a)), on the centerline of the wake in the middle of the vertical gap between two successive cylinders, (point (b)) and at point (c) located at $l \mathbf{e}_{x}$ from point (a). We recall that the critical Reynolds number for this structure is $R e_{d c}=100.82 \pm 2.5$ $\left(R e_{c}^{*}=17250 \pm 500\right)$. In parallel, a spectral analysis is carried out in terms of the Strouhal number given by $S t=\frac{f l}{v_{r e f}}=\frac{\mu_{\beta} f}{l\left|\nabla\left\langle p_{\beta}\right\rangle^{\beta}\right|}, f$ being the frequency of oscillations of $v_{\beta y}^{*}$ once this velocity has been Fourier transformed using an FFT algorithm. The analysis is made for dimensionless times ranging from $1.22 \times 10^{4}$ to $2.455 \times 10^{4}\left(\Delta t^{*}=0.025\right)$.

At the limit of steady flow $\left(R e_{d}=98.32, R e^{*}=16750\right)$, permanent flow is obtained from $t^{*} \approx 0.25 \times 10^{4}$ on. The spectral analysis on point (a) shows a zero single dominant frequency (see Fig. 14a). At the onset of unsteady flow $\left(R e_{d c}=103.32, R e^{*}=17750\right)$ for which a permanent behavior is reached at $t^{*} \approx 1.5 \times 10^{4}$, the analysis at point (a) shows a quasi-periodic nature of the flow, characterized by four distinct frequencies. The two first ones correspond to Strouhal numbers $S t \approx 0.01$ while the two largest ones, associated to modes of smaller amplitudes, are characterized by Strouhal numbers $S t \approx 0.03$ (see Fig. 14b-d). These results contrast with those obtained for flow around an isolated square cylinder [50] for which the bifurcation is characterized by a periodic unsteady flow (i.e. a unique non-zero frequency). A similar behavior was pointed out by Dybbs et al. [66] for flow around spheres and by Ghaddar et al. [51] for flow around parallel circular cylinders. In this latter case, vortex shedding on a periodic structure was observed to be composed of a traveling wave made of a combination of modes of distinct periods and amplitudes.

Spectra at points (b) and (c) for $R e_{d c}=103.32\left(R e^{*}=17750\right)($ Figs. 14c-d) show that the same frequency content is observed on adjacent geometrical unit cells. This is a clear indication that the non-periodic flow at scale $l$ observed on Fig. 9b is simply due to a phase shift from one geometrical unit cell to another.

For a larger Reynolds number $\left(R e_{d c}=129.46, R e^{*}=25000\right.$, see Fig. 14e), characteristic frequencies observed at the Hopf bifurcation persist while amplitudes of the corresponding modes increase. Moreover, as the Reynolds number increases, linear combinations of the four frequencies are appearing. Here, it must be emphasized that permanent flow is reached faster as the Reynolds number increases when simulation is initiated with the fluid at rest.

Finally, for a Reynolds number $R e_{d} \approx 200$, a transition from quasi-periodic flow to a frequency-locking mode is observed. This is characterized by the apparition of new observable spectral peaks that are non linear interactions of the four frequencies observed at the onset of unsteady flow.

\subsubsection{Influence of $\nabla\left\langle p_{\beta}^{*}\right\rangle^{\beta}$ orientation}

The influence of the orientation of the dimensionless gradient of the average pressure gradient $\nabla\left\langle p_{\beta}^{*}\right\rangle^{\beta}$ on $R e_{d c}$ in the $O S$ for $\epsilon=75 \%$ is investigated in this section using $\nabla\left\langle p_{\beta}^{*}\right\rangle^{\beta}$ oriented $45^{\circ}$ with respect to the two principal directions of the structure, i.e. $\nabla\left\langle p_{\beta}^{*}\right\rangle^{\beta}=\left(\mathbf{e}_{x}+\mathbf{e}_{y}\right) / \sqrt{2}$. Two REVs, respectively 
made of $1 \times 1$ and $3 \times 3$ geometrical unit cells, were employed along with periodic boundary conditions to determine $R e_{d c}$. In contrast with the case where $\nabla\left\langle p_{\beta}^{*}\right\rangle^{\beta}=\mathbf{e}_{x}$, no impact of the REV size was noticed as $R e_{d c}=29.7 \pm 0.6\left(R e_{c}^{*}=12500 \pm 500\right)$ was found in both cases. This observation may be explained by the fact that in the present case, oscillating eddies when unsteadiness appears, do not develop across the boundaries where periodicity is imposed. The critical Reynolds number value is more than three times smaller than that obtained with $\nabla\left\langle p_{\beta}^{*}\right\rangle^{\beta}=\mathbf{e}_{x}\left(R e_{d c}=100.82 \pm 2.5, R e_{c}^{*}=17250 \pm 500\right)$. As suggested by the flow structure illustrated in Fig. 15, this much smaller value of $R e_{d c}$ can be attributed to the absence of preferential channels yielding significantly more tortuous streamlines that cover the entire domain and for which symmetry can be more easily broken, triggering the bifurcation to unsteady state.

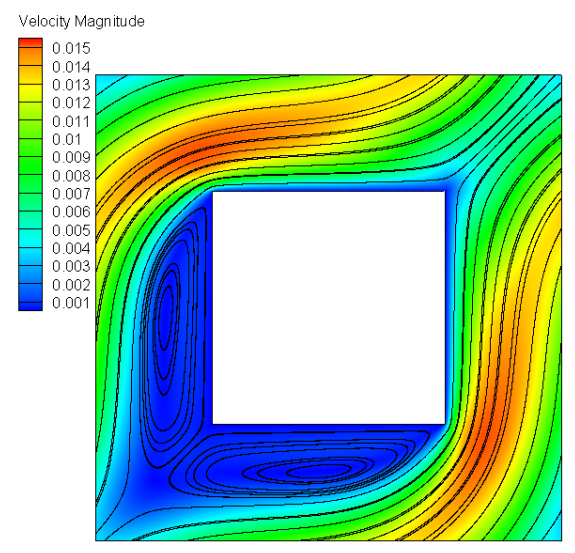

(a) Steady flow. $R e_{d}=29.1\left(R e^{*}=12000\right)$.

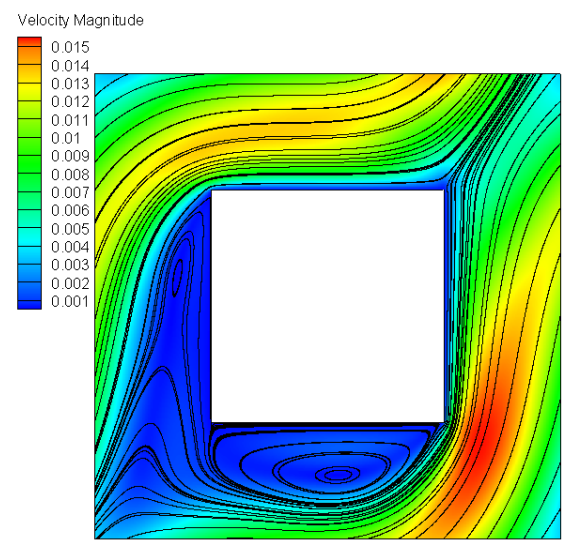

(c) Unsteady flow. $R e_{d}=30.32\left(R e^{*}=\right.$ 13000). $t^{*}=27760$.

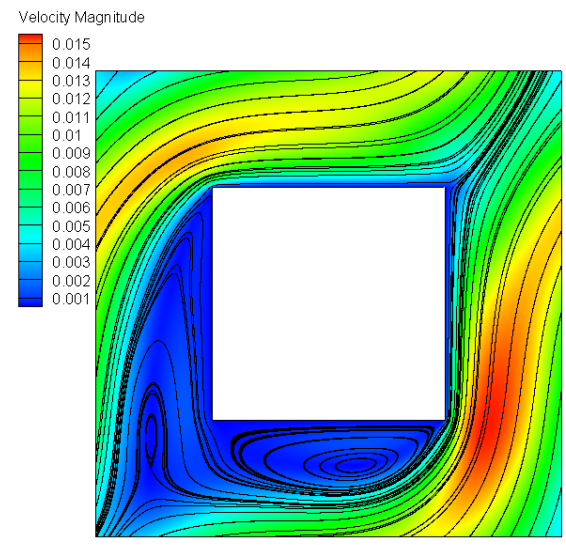

(b) Unsteady flow. $R e_{d}=30.32\left(R e^{*}=\right.$ 13000). $t^{*}=27740$.

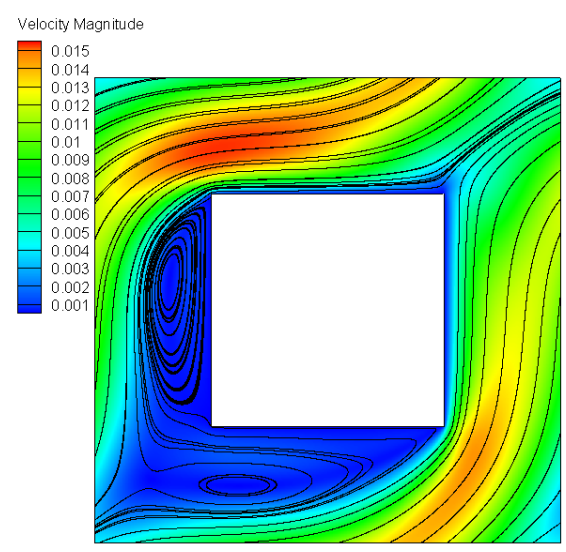

(d) Unsteady flow. $R e_{d}=30.32\left(R e^{*}=\right.$ 13000). $t^{*}=27800$.

Figure 15: Streamlines and velocity magnitude color maps. OS. REV composed of $1 \times 1$ geometrical unit cell. $\epsilon=75 \%$. $\nabla\left\langle p_{\beta}^{*}\right\rangle^{\beta}=\left(\mathbf{e}_{x}+\mathbf{e}_{y}\right) / \sqrt{2}$. 


\subsection{Disordered structures}

The purpose is now to analyze the impact of structural disorder on the critical Reynolds number. Here, disordered structures $(D S)$ correspond to arrays made of $n \times n$ parallel cylinders of square cross section having all the same size, each center of individual square inclusion being randomly placed in the original unit cell of the corresponding ordered structure following a uniform distribution (see Fig. 16). To avoid too close adjacent cylinders, the random placement was performed in a subdomain of size $0.85 l$. The final disordered domain is obtained by reassembling $n \times n$ random cells of edge size $l$ obtained from this process (see [23]).

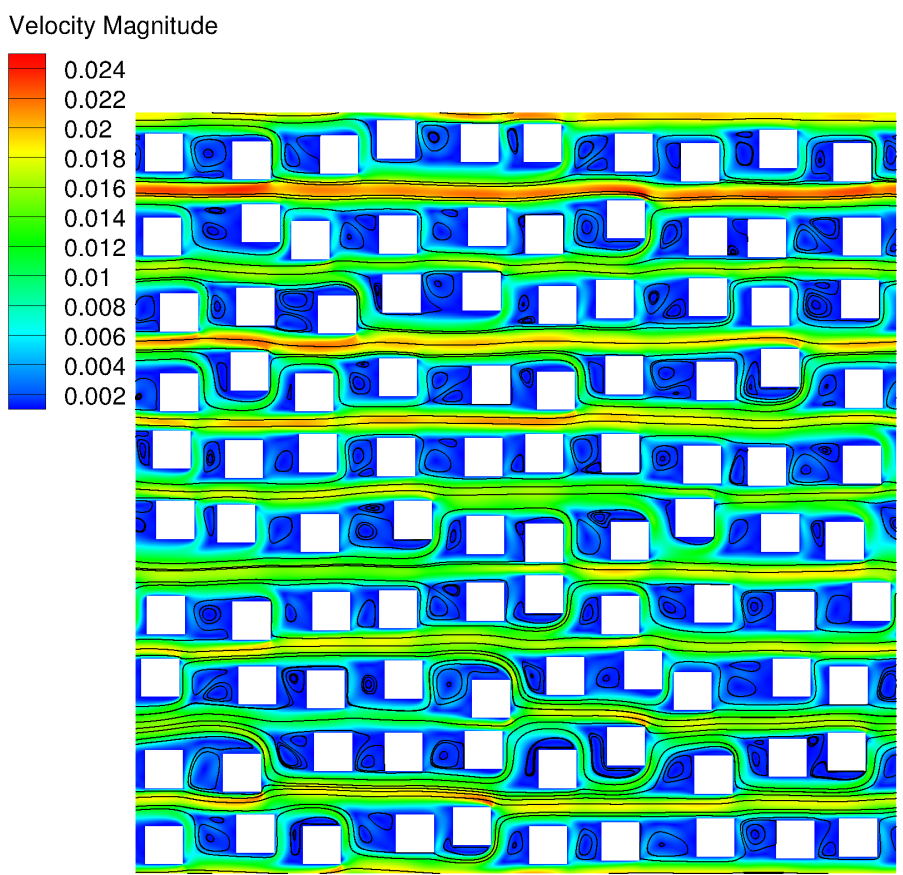

Figure 16: Streamlines and velocity magnitude color map. DS. $\epsilon=75 \% . \nabla\left\langle p_{\beta}^{*}\right\rangle^{\beta}=\mathbf{e}_{x}$. Limit of steady flow, $R e_{d}=85.92$ $\left(R e^{*}=30000\right)$.

The dependence of $R e_{d c}$ on porosity was investigated in the range $36 \% \leq \epsilon \leq 75 \%$, keeping the pressure gradient oriented along $\mathbf{e}_{x}$. For these structures, computational domains with $n=10$ were considered and this choice was guided by an analysis of inertial regimes performed on similar structures [23]. Since the purpose is to estimate the Reynolds number at the bifurcation expected on an infinite medium having the same statistical structural properties, an ergodic assumption was employed. The computation of $R e_{d c}$ was carried out on five different realizations of the same structure, a procedure that remains computationally tractable although each realization was discretized with a grid-block number ranging from roughly $7.7 \times 10^{5}$ for $\epsilon=36 \%$ to approximately $1.6 \times 10^{6}$ for $\epsilon=75 \%$. The value of $R e_{d c}$ is estimated from the arithmetic mean of the values obtained for the five different realizations. 


\begin{tabular}{ccccc}
\hline \hline$\epsilon(\%)$ & $R e_{d c}($ mean $)$ & $\sigma_{R e}$ & $k^{*}($ mean $)$ & $\sigma_{k}$ \\
\hline 36 & $331.53 \pm 6.21$ & 27.7 & $72.03 \times 10^{-5}$ & $74.11 \times 10^{-7}$ \\
55.11 & $156.71 \pm 5.68$ & 12.47 & $34.47 \times 10^{-4}$ & $46.51 \times 10^{-6}$ \\
75 & $83.51 \pm 2.45$ & 4.69 & $13.15 \times 10^{-3}$ & $25.38 \times 10^{-5}$
\end{tabular}

Table 1: Computational results on the mean values and standard deviations of $R e_{d c}$ and $k^{*}$ obtained on five different realizations of $D S$ and three values of the porosity.

In Table 1, we have reported the results on $R e_{d c}$ and $k^{*}$ (mean values and standard deviations $\sigma_{R e}$ and $\sigma_{k}$ ) for the three values of $\epsilon$. Fitting curves are such that $a_{0}=127.86, b_{0}=-0.234, c_{0}=0.59$ (see Eq. (15)) whereas $a_{1}=0.0137, b_{1}=3.07, c_{1}=0.707$ (see Eq. (16)), the correlation coefficient being $R^{2}=0.9999$ over the interval $0.36 \lesssim \epsilon \lesssim 0.75$ for both quantities.

While the Reynolds number at the bifurcation is decreasing with increasing $\epsilon$, as was observed on $O S$, it must be noted that the range of variation of $R e_{d c}$ is much narrower compared to the $O S$. This is further illustrated in Fig. 17 where our results on $R e_{d c}$ are represented versus $\epsilon$ for both $O S$ and $D S$. More precisely, for $\epsilon$ close to unity, $R e_{d c}$ is weakly modified by the presence of disorder, while the bifurcation on $D S$ occurs for much smaller Reynolds numbers when porosity decreases. The origin of this strong modification for small values of $\epsilon$ lies in the local enlargements and constrictions together with obstacles misalignment that create tortuous channels as a result of disorder. Misalignment breaks flow symmetry and forces local flow separation as illustrated in Fig. 18. Enlargements in the direction perpendicular to $\nabla\left\langle p_{\beta}^{*}\right\rangle^{\beta}$ favor eddies generation at significantly smaller Reynolds numbers that disturb the flow in the tortuous channels oriented along $\nabla\left\langle p_{\beta}^{*}\right\rangle^{\beta}$ contributing to early bifurcation towards unsteadiness. When cylinders are far enough apart (i.e. for large enough values of the porosity), the influence of all these effects is much less significant as the relative impact of disorder on the sizes of the gaps is smaller, explaining why $R e_{d c}$ remains quasi unmodified when disorder is present in this range of $\epsilon$. 


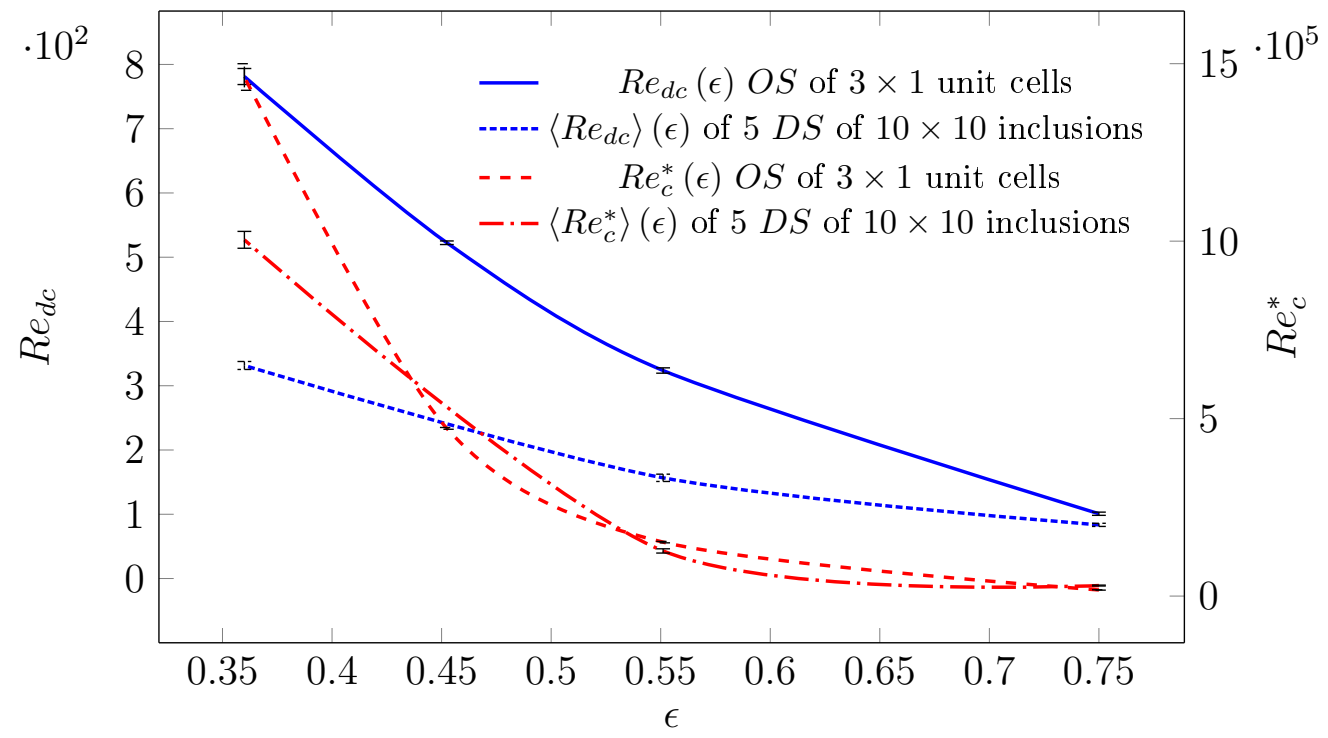

Figure 17: Dependence of the critical Reynolds number $R e_{d c}$ (or $R e_{c}^{*}$ ) on $\epsilon$ and sigmoidal fit curves (see text). Vertical bars materialize the upper and lower bounds of $R e_{d}$ (or $R e^{*}$ ) at which unsteady and steady flows are respectively observed. Ordered $(O S)$ and disordered $(D S)$ structures. $\nabla\left\langle p_{\beta}^{*}\right\rangle^{\beta}=\mathbf{e}_{x}$.

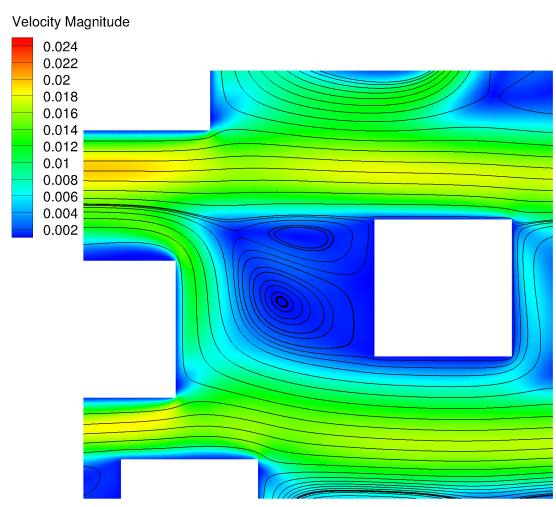

Figure 18: Close-up view of streamlines and velocity magnitude color map extracted from Fig. 16. DS. $\epsilon=75 \% . \nabla\left\langle p_{\beta}^{*}\right\rangle^{\beta}=$ $\mathbf{e}_{x}$. Limit of steady flow, $R e_{d}=85.92\left(R e^{*}=30000\right)$.

\section{Conclusions}

Direct Numerical Simulations of one-phase incompressible flow on $2 D$ periodic structures composed of cylinders of square cross section as model porous materials have been carried out. Special attention has been paid to computational accuracy (grid-block size and solvers tolerances) in order to investigate the occurrence of unsteadiness corresponding to the first Hopf bifurcation. The bifurcation was characterized by a critical Reynolds number $R e_{d c}$ at the center of a Reynolds number interval whose lower and upper bounds are 
respectively the largest and smallest values for which steady and unsteady flow is observed. The overall procedure was validated by successful comparisons of $R e_{d c}$ with reported values on several configurations namely transverse flow over an isolated cylinder of square cross section and cylinders of circular cross section arranged on a square pattern.

Our analysis, focused on ordered $(O S)$ and disordered $(D S)$ square patterns of parallel cylinders of square cross section, highlighted several important results.

First, it was shown that, for $O S$ and a macroscopic pressure gradient along one of the lattice axes, the determination of $R e_{d c}$ requires a computational domain (i.e. a Representative Elementary Volume (REV) of the structure) larger than the geometrical periodic unit cell in the flow direction while employing periodic boundary conditions on velocity and pressure deviation. This is of particular importance when the porosity of the structure under concern is larger than $\epsilon \sim 45 \%$. The critical Reynolds number was determined over a wide range of porosities showing that $R e_{d c}$ strongly decreases with $\epsilon$. Even if this behaviour is quantitatively highly structure-dependent, it can certainly be generalized to any other kind of geometries and in 3D. The flow analysis highlighted that the bifurcation is triggered by the oscillation of asymmetric vortices which take place between two successive cylinders along the flow direction. A spectral analysis on the velocity component orthogonal to the applied macroscopic pressure gradient indicates that vortices oscillation at the bifurcation is quasi-periodic, characterized by two pairs of frequencies, the two frequencies in each pair being close to each others. These frequencies persist for post-bifurcation Reynolds numbers up to a frequency locking mode that occurs at large enough Reynolds numbers.

In a second step, the impact of the macroscopic pressure gradient orientation relative to the lattice axes was illustrated on the $O S$ indicating that when it is not aligned with the two principal directions of the structure, $R e_{d c}$ is strongly decreased. This results from much more tortuous streamlines induced by the absence of preferential flow channels compared to the case where the pressure gradient is along one of the lattice axes. For a macroscopic pressure gradient oriented at $45^{\circ}$ with respect to the lattice axes, no influence of the REV size was observed.

Computations carried out on $D S$ indicated that bifurcation occurs at much smaller Reynolds numbers compared to $O S$ having the same porosity. The reduction is much more significant as $\epsilon$ decreases. The physical explanation lies in the occurrence of local enlargements, together with misalignment introduced by disorder leading to much more tortuous streamlines, local flow separation, as well as early vortices symmetry breaking and oscillation.

For both $O S$ and $D S$, the Reynolds number at the Hopf bifurcation was accurately correlated to the porosity according to sigmoidal functions over a wide range of $\epsilon$. These relationships are valid for model porous structures made of parallel cylinders that are however representative of many real configurations like networks of streets and buildings or forests for which many different flow, heat and/or mass transfer-related 
problems are of major concern. As expected, it clearly appears that disorder favors mixing and could be introduced to improve this mechanism as a less energy demanding alternative to increasing flow rate while keeping order.

Even if significant effect on the critical Reynolds number was pointed out when weak disorder is introduced, a thorough study would be necessary when stronger disorder is considered, in terms of shapes, size and spatial distributions of the solid phase. Additional work is also needed to extend the analysis to more realistic porous structures using detailed pore-scale images.

\section{Acknowledgments}

Computational resources for this study were provided by the computing facility MCIA (Mésocentre de Calcul Intensif Aquitain) of the University of Bordeaux which is gratefully acknowledged.

\section{References}

[1] F. J. Valdés-Parada, J. R. Varela, J. Alvarez-Ramirez, Upscaling pollutant dispersion in the Mexico City Metropolitan Area, Physica A 391 (3) (2012) 606-615.

[2] Y. Tominaga, A. Mochida, R. Yoshie, H. Kataoka, T. Nozu, M. Yoshikawa, T. Shirasawa, AIJ guidelines for practical applications of CFD to pedestrian wind environment around buildings, J. Wind Eng. Ind. Aerodyn. 96 (10) (2008) 1749-1761.

[3] A. Sohankar, C. Norberg, L. Davidson, Simulation of three-dimensional flow around a square cylinder at moderate Reynolds numbers, Phys. Fluids 11 (2) (1999) 288-306.

[4] J. D. Wilson, T. K. Flesch, Wind and remnant tree sway in forest cutblocks. III. A windflow model to diagnose spatial variation, Agric. For. Meteorol. 93 (4) (1999) 259-282.

[5] M. D. Novak, J. S. Warland, A. L. Orchansky, R. Ketler, S. Green, Wind tunnel and field measurements of turbulent flow in forests. Part I: uniformly thinned stands, Boundary Layer Meteorol. 95 (3) (2000) $457-495$.

[6] B. Ruck, E. Adams, Fluid mechanical aspects of the pollutant transport to coniferous trees, Boundary Layer Meteorol. 56 (1-2) (1991) 163-195.

[7] C. Gromke, B. Ruck, Aerodynamic modelling of trees for small-scale wind tunnel studies, Forestry 81 (3) (2008) 243-258.

[8] H. Darcy, Les fontaines publiques de la ville de Dijon: exposition et application, Victor Dalmont, 1856. 
[9] S. Irmay, On the theoretical derivation of Darcy and Forchheimer formulas, EOS, Trans. Am. Geophys.

[10] S. P. Neuman, Theoretical derivation of Darcy's law, Acta Mech. 25 (3-4) (1977) 153-170.

[11] S. Whitaker, Flow in porous media I: A theoretical derivation of Darcy's law, Transp. Porous Media 1 (1) (1986) 3-25.

[12] P. Forchheimer, Wasserbewgung durch boden, Vereines deutscher ingnieure XXXXV (45) (1901) 1782-

[13] V. D. Cvetkovic, A continuum approach to high velocity flow in a porous medium, Transp. Porous Media 1 (1) (1986) 63-97.

[14] S. M. Hassanizadeh, W. G. Gray, High velocity flow in porous media, Transp. Porous Media 2 (6) (1987) $521-531$.

[15] H. Ma, D. W. Ruth, The microscopic analysis of high Forchheimer number flow in porous media, Transp. Porous Media 13 (2) (1993) 139-160.

[16] S. Whitaker, The Forchheimer equation: a theoretical development, Transp. Porous Media 25 (1) (1996) $27-61$.

[17] T. Giorgi, Derivation of the Forchheimer law via matched asymptotic expansions, Transp. Porous Media 525

[18] Z. Chen, S. L. Lyons, G. Qin, Derivation of the Forchheimer law via homogenization, Transp. Porous Media 44 (2) (2001) 325-335.

[19] J. C. Wodie, T. Levy, Correction non linéaire de la loi de Darcy, C. R. Acad. Sci. Série II 312 (3) (1991) $157-161$.

[20] C. C. Mei, J. L. Auriault, The effect of weak inertia on flow through a porous medium, J. Fluid Mech. 222 (1991) 647-663.

[21] M. Rasoloarijaona, J. L. Auriault, Nonlinear seepage flow through a rigid porous medium, Eur. J. Mech. B. Fluids 13 (2) (1994) 177-195.

[22] E. Skjetne, J. L. Auriault, New insights on steady, non-linear flow in porous media, Eur. J. Mech. B. Fluids 18 (1) (1999) 131-145.

[23] D. Lasseux, A. A. Arani, A. Ahmadi, On the stationary macroscopic inertial effects for one phase flow in ordered and disordered porous media, Phys. Fluids 23 (2011) 073103. 
[24] M. Agnaou, D. Lasseux, A. Ahmadi, Inertial flow in porous media: A numerical investigation on model structures, in: Proceedings of the 5th International Conference on Porous Media, 2013. Comput. Methods Appl. Mech. Engrg. 195 (2006) 6046-6058.

[27] M. J. de Lemos, M. H. Pedras, Recent mathematical models for turbulent flow in saturated rigid porous

[28] B. V. Antohe, J. L. Lage, A general two-equation macroscopic turbulence model for incompressible flow in porous media, Int. J. Heat Mass Transfer 40 (13) (1997) 3013-3024.

[29] A. Nakayama, F. Kuwahara, A macroscopic turbulence model for flow in a porous medium, J. Fluids Eng. 121 (2) (1999) 427-433.

[30] C. Soulaine, M. Quintard, On the use of a Darcy-Forchheimer like model for a macro-scale description of turbulence in porous media and its application to structured packings, Int. J. Heat Mass Transfer 74 (2014) 88-100.

[31] B. N. Rajani, A. Kandasamy, S. Majumdar, Numerical simulation of laminar flow past a circular cylinder, Appl. Math. Modell. 33 (3) (2009) 1228-1247.

[32] S. Dong, G. E. Karniadakis, DNS of flow past a stationary and oscillating cylinder at Re=10000, J. Fluids Struct. 20 (4) (2005) 519-531.

[33] A. Sohankar, L. Davidson, C. Norberg, Numerical simulation of unsteady flow around a square twodimensional cylinder, in: Proc. 12-th AFMC, 1995, pp. 517-520.

[34] A. Sohankar, C. Norberg, L. Davidson, Numerical simulation of flow past a square cylinder, in: Proc. of FEDSM99 3rd ASME/JSME Joint Fluids Engineering Conference. July, 1999, pp. 18-23.

[35] D. H. Yoon, K. S. Yang, C. B. Choi, Flow past a square cylinder with an angle of incidence, Phys. Fluids 22 (4) (2010) 043603.

[36] M. Cheng, P. M. Moretti, Experimental study of the flow field downstream of a single tube row, Exp. Therm Fluid Sci. 1 (1) (1988) 69-74.

[37] J. Mizushima, T. Akinaga, Vortex shedding from a row of square bars, Fluid Dyn. Res. 32 (4) (2003) 179-191. 
[38] S. R. Kumar, A. Sharma, A. Agrawal, Simulation of flow around a row of square cylinders, J. Fluid Mech. 606 (2008) 369-397.

[39] D. L. Koch, A. J. Ladd, Moderate Reynolds number flows through periodic and random arrays of aligned cylinders, J. Fluid Mech. 349 (1997) 31-66.

[40] J. Wu, S. Yin, Non-Darcy seepage and stopped floor water inrush prevention, Progress in Mine Safety Science and Engineering II (2014) 163.

[41] E. Sanchez-Palencia, Non Homogeneous Media and Vibration Theory, Lecture Notes in Physics, Springer, 1980.

[42] S. Whitaker, The method of volume averaging, Theory and Applications of Transport in Porous Media, Kluwer Academic, Dordrecht, The Netherlands, 1999.

[43] W. G. Gray, A derivation of the equations for multi-phase transport, Chem. Eng. Sci. 30 (2) (1975) 229-233.

[44] M. Zuzovsky, P. M. Adler, H. Brenner, Spatially periodic suspensions of convex particles in linear shear flows. III. Dilute arrays of spheres suspended in Newtonian fluids, Phys. Fluids 26 (7) (1983) 1714-1723.

[45] S. Rojas, J. Koplik, Nonlinear flow in porous media, Phys. Rev. E 58(4) (1998) 4476-4782.

[46] T. D. Papathanasiou, B. Markicevic, E. D. Dendy, A computational evaluation of the Ergun and Forchheimer equations for fibrous porous media, Phys. Fluids 13 (10) (2001) 2795-2804.

[47] Foundation OpenFoam, Openfoam the open source CFD toolbox user guide version 2.3.0 (February $20142014)$.

[48] R. I. Issa, Solution of the implicitly discretized fluid flow equations by operator-splitting, J. Comput. Phys. 62 (1985) 40-65.

[49] X. H. Wang, W. F. Zhu, Z. Y. He, Numerical estimation of the critical Reynolds number for flow past one square cylinder with symmetric geometry boundary condition, in: New Trends in Fluid Mechanics Research, Springer, 2009, pp. 76-76.

[50] A. K. Saha, K. Muralidhar, G. Biswas, Transition and chaos in two-dimensional flow past a square cylinder, J. Eng. Mech. 126 (5) (2000) 523-532.

[51] C. K. Ghaddar, On the permeability of unidirectional fibrous media: a parallel computational approach, Phys. Fluids 7 (11) (1995) 2563-2586. 
[64] K. Pearson, Note on regression and inheritance in the case of two parents, Proc. R. Soc. London 58 (347352) (1895) 240-242.

[53] W. K. Anderson, J. T. Batina, Accurate solutions, parameter studies and comparisons for the euler and potential flow equations, in: AGARD, Validation of Computational Fluid Dynamics., Vol. 1, 1988.

[54] C. L. Rumsey, M. D. Sanetrik, R. T. Biedron, N. D. Melson, E. B. Parlette, Efficiency and accuracy of time-accurate turbulent Navier-Stokes computations, Comput. Fluids 25 (2) (1996) 217-236.

[55] S. V. Patankar, D. B. Spalding, A calculation procedure for heat, mass and momentum transfer in three-dimensional parabolic flows, Int. J. Heat Mass Transfer 15 (10) (1972) 1787-1806.

[56] J. P. V. Doormaal, G. D. Raithby, An evaluation of the segregated approach for predicting incompressible fluid flows, ASME paper NHTC (85-HT) (1985) 9.

[57] L. Zhang, DNS study of flow over periodic and random distribution of cylinders and spheres, Ph.D. thesis, University of Illinois at Urbana-Champaign (2008).

[58] J. A. R. Vázquez, A computational fluid dynamics investigation of turbulent swirling burners, Ph.D. thesis, Universidad de Zaragoza, Instituto Universitario de Investigación Mixto CIRCE (2012).

[59] A. Lankadasu, S. Vengadesan, Onset of vortex shedding in planar shear flow past a square cylinder, Int. J. Heat Fluid Flow 29 (4) (2008) 1054-1059.

[60] A. Sohankar, C. Norberg, L. Davidson, Low-Reynolds-number flow around a square cylinder at incidence: study of blockage, onset of vortex shedding and outlet boundary condition, Int. J. Numer. Methods Fluids 26 (1) (1998) 39-56.

[61] J. G. I. Hellstrom, P. J. P. Jonsson, T. S. Lundstrom, Laminar and turbulent flow through an array of cylinders, J. Porous Media 13 (12) (2010) 1073-1085.

[62] F. Kuwahara, T. Yamane, A. Nakayama, Large eddy simulation of turbulent flow in porous media, Int. Commun. Heat Mass Transfer 33 (4) (2006) 411-418.

[63] P. S. Casas, Á. Jorba, Hopf bifurcations to quasi-periodic solutions for the two-dimensional plane poiseuille flow, Commun. Nonlinear Sci. Numer. Simul. 17 (7) (2012) 2864-2882. 
[65] J. L. Rodgers, W. A. Nicewander, Thirteen ways to look at the correlation coefficient, Am. Stat. 42 (1) (1988) 59-66.

${ }_{625}$ [66] A. Dybbs, R. V. Edwards, A new look at porous media fluid mechanics - Darcy to turbulent, in: Fundamentals of Transport Phenomena in Porous Media, Springer, 1984, pp. 199-256. 